\title{
D-HMBC versus LR-HSQMBC: Which experiment provides theoretically and experimentally the best results?
}

\author{
Peter Bigler*, Julien Furrer*
}

\section{Departement für Chemie und Biochemie, Universität Bern,} Freiestrasse 3, CH-3012 Bern, Switzerland

\section{*biglermeier@bluewin.ch (PB)}

\section{*julien.furrer@dcb.unibe.ch (JF)}

Abstract The Long-Range Heteronuclear Single Quantum Multiple Bond Correlation (LR-HSQMBC) experiment is the experiment of choice for visualizing heteronuclear long-range coupling interactions ${ }^{n} J_{C H}$ across $4-6$-bonds and is experimentally superior to the Decoupled Heteronuclear Multiple-Bond Correlation (D-HMBC) experiment. Yet, the exact reasons have not been fully understood and established. On the basis of our recent investigation of the non-refocused variants LR-HSQC and HMBC, we have extended a $\mathrm{JHH}^{\prime}$-dedicated investigation to the D-HMBC- and LRHSQMBC experiments. Unlike the non-refocused variants, the influence of homonuclear couplings $\mathrm{JHH}^{\prime}$ on the intensity of long-range ${ }^{n} J_{\mathrm{CH}}$ cross-peaks is not easily predictable and may be summarized as follows: (i) irrespective of the magnitude and number of $\mathrm{JHH}$ interactions long-range ${ }^{n} \mathrm{~J}_{\mathrm{CH}}$ cross-peaks are more intense in D-HMBC spectra as long as the evolution delay $\Delta$ is not too large, since in contrast to LR-HSQMBC no $\mathrm{JHH}_{\mathrm{H}}$-caused intensity zeroes will occur. (ii) If $\mathrm{JHH}_{\mathrm{H}}$ is small and $\Delta$ large, the intensity of cross peaks in D-HMBC spectra may be weakened or may even vanish at $\Delta=(0.25+0.5 k) / \mathrm{JHH}^{\prime}$, while for the LR-HSQMBC this unwanted effect occurs at $\Delta=k+0.5 / \mathrm{J}_{\mathrm{HH}}$. Consequently, when $\Delta$ is adjusted to visualize weak $n J_{\mathrm{CH}}$ long-range correlations, our findings corroborate that there are potentially more cross-peaks expected to show up in a LR-HSQMBC spectrum compared to a D-

This article has been accepted for publication and undergone full peer review but has not been through the copyediting, typesetting, pagination and proofreading process which may lead to differences between this version and the Version of Record. Please cite this article as doi: $10.1002 / m r c .4810$ 
HMBC spectrum. This has been indeed noticed experimentally, even though the intensity of a many long-range ${ }^{n} J_{C H}$ cross-peaks may still be higher in the spectra of the D-HMBC experiment correspondingly adjusted for detecting weak ${ }^{n} J_{C H}$ correlations.

Keywords: NMR, ${ }^{1} \mathrm{H},{ }^{13} \mathrm{C}, \mathrm{D}-\mathrm{HMBC}$, LR-HSQMBC, homonuclear couplings, product operator, cross peak intensity

\section{Introduction}

Heteronuclear long-range correlation experiments are essential for establishing molecular structures based on corresponding spin-spin coupling networks. For scientists, these experiments actually represent the sole possibility for connecting molecular fragments via non-protonated carbons or heteroatoms through $J$-couplings. Currently, there are a plethora of proton-detected methods available for long-range heteronuclear shift correlation. ${ }^{[1-3]}$ The oldest and probably still the most widely used long-range heteronuclear shift correlation experiment is the basic HMBC experiment described in 1986 by Bax and Summers. ${ }^{[4]}$ This pulse sequence consists of only a few RF pulses, making it not only very robust in terms of RF inhomogeneities but also the most sensitive. ${ }^{[5]}$

HMBC experiments, typically optimized for $\mathrm{C}, \mathrm{H}$-long-range couplings ${ }^{n} J_{\mathrm{CH}}$ in the range of $6-10 \mathrm{~Hz}$, provide access primarily to ${ }^{3} \mathrm{~J}_{\mathrm{CH}}$ correlations, while the often smaller ${ }^{2} \mathrm{JCH}_{\mathrm{CH}}$ and ${ }^{4} \mathrm{~J}_{\mathrm{CH}}$ couplings will give rise to weak or even non-visible correlation signals in HMBC spectra. Sporadically, especially for rigid cyclic and polycyclic molecules, long-range correlations across up to six bonds $\left({ }^{6} \mathrm{~J}_{\mathrm{CH}}\right)$ are observed. ${ }^{[6-8]}$ Many molecules are however proton deficient, and for these compounds there is a crucial need for detecting and measuring ${ }^{4} \mathrm{~J} C H$ and sometimes still longer range connectivities to fully establish or confirm the molecular structure. The 1,nADEQUATE experiment originally developed by Griesinger at al. ${ }^{[9,10]}$ and improved by Martin et al. ${ }^{[11]}$ provides this capability via ${ }^{3} \mathrm{JcC}_{\mathrm{C}}$ (equivalent to ${ }^{4} \mathrm{~J}_{\mathrm{CH}}$ ) correlations. However, due to the low abundance of doubly ${ }^{13} \mathrm{C}$-labelled isotopomers, the sensitivity of this experiment is excessively low, and its application requires access to cryogenic NMR probe capabilities if the technique is to be applied in the low 
milligram range or to work with expensive ${ }^{13} \mathrm{C}$-labeled compounds. ${ }^{[12]}$ An alternative strategy is to optimize the HMBC experiment for a small long-range coupling in the range of $2-4 \mathrm{~Hz}$. However, this induces a considerable loss in sensitivity of crosspeaks due to (i) relaxation losses, and (ii) mutual cancelation of antiphase signals with small couplings. ${ }^{[6]}$

Several years after the introduction of the HMBC experiment a decoupled variant, D-HMBC, ${ }^{[13]}$ was introduced. Due to an additional long-range coupling evolution delay, heteronuclear antiphase proton coherences will refocus into inphase coherences. This avoids not only this unwanted mutual cancelation with small ${ }^{n} J_{\mathrm{CH}}$ couplings but also allows ${ }^{13} \mathrm{C}$ decoupling to be applied during the acquisition time. It has been proven that for these reasons and with small ${ }^{n} J_{C H}$ couplings a higher sensitivity may be achieved with D-HMBC compared to the basic HMBC experiment despite additional relaxation losses with the prolonged experiment and losses caused by a second non-optimally adjusted long-range coupling evolution delays. ${ }^{[13]}$ Still, the D-HMBC has never enjoyed the popularity of the HMBC experiment, although it can occasionally provide more ${ }^{n} J_{C H}$ correlations than the classical HMBC. ${ }^{[14]}$

Long-Range Heteronuclear Single Quantum Correlation (LR-HSQC) experiments may be applied as an alternative to HMBC experiments for detecting long-range correlations, but these experiments are actually almost exclusively employed for the measurements of long-range coupling constants. ${ }^{[15-18]}$ Recently, we have shown both theoretically and experimentally that the classical sensitivityenhanced HMBC experiment (HMBC-SE) is superior to the LR-HSQC for detecting long-range correlations for the two main reasons: (i) long-range cross peaks are generally more intense in $\mathrm{HMBC}$ spectra, and (ii) the intensity of the long-range cross peaks in LR-HSQC spectra is influenced not only by the size of ${ }^{n} J_{C H}$ but additionally and in an unwanted way by the magnitude and number of passive homonuclear proton-proton couplings $\mathrm{JHH}^{\prime} \cdot{ }^{[19]}$

A few years ago, Williamson et al. have introduced the LR-HSQMBC experiment, which is a refocused and decoupled variant of the G-BIRD ${ }^{r,}{ }_{-}-H_{S Q M B C}$ pulse sequence ${ }^{[20,21]}$ optimized for the observation of weak long-range correlations. ${ }^{[6]}$ Compared to the HMBC sequences optimized for very small coupling constants and similar to the D-HMBC sequence, the LR-HSQMBC creates in-phase ${ }^{n} J_{C H}$ correlations. This avoids the mutual cancelation of antiphase responses and allows 
the application of ${ }^{13} \mathrm{C}$ decoupling during the acquisition time. As shown by Williamson et al., the LR-HSQMBC experiment overcomes the typical ${ }^{2,3} \mathrm{~J}_{\mathrm{CH}}$ limitation of $\mathrm{HMBC}$ and extends the range of long-range correlations to 4-, 5-, and even 6bond long-range ${ }^{n} J_{\mathrm{CH}}$ heteronuclear couplings. ${ }^{6]}$

The authors reported that the LR-HSQMBC experiment performs experimentally much better than the $\mathrm{HMBC}$ and $\mathrm{D}-\mathrm{HMBC}$, not only for very weak responses, but also for the full range of ${ }^{n} J_{C H}$ responses. ${ }^{[6]}$ For strychnine, the $2 \mathrm{~Hz}$ optimized LR-HSQMBC provided a total of 68 weak long-range correlations $\left(>{ }^{3} \mathrm{~J} C \mathrm{H}\right)$. There were 14 additional weak long-range responses compared to the $2 \mathrm{~Hz}$ optimized HMBC, and even 24 additional very weak responses compared to the 2 $\mathrm{Hz}$ optimized D-HMBC. The LR-HSQMBC also revealed a total of $160 \mathrm{n}_{\mathrm{CH}}$ correlations, while 142 correlations were observed using the $2 \mathrm{~Hz} \mathrm{HMBC}$, and only 115 correlations in the $2 \mathrm{~Hz}$ D-HMBC data. ${ }^{[6]}$ Importantly, many of the very weak correlations that can be observed in an LR-HSQMBC spectrum may not be observed at all when less than $512 t_{1}$ increments are acquired in the $\mathrm{F} 1$ dimension. In many instances, weak ${ }^{5} \mathrm{~J}_{\mathrm{CH}}$ and ${ }^{6} \mathrm{~J}_{\mathrm{CH}}$ correlations are not observed until 640 or even $768 t_{1}$ increments have been acquired. ${ }^{6,22]}$

In an effort for better understanding the origin of the reported superiority of the LR-HSQMBC for detecting long-range and very long-range ${ }^{n} J_{C H}$ couplings, we embarked on a detailed theoretical and experimental comparative study of the DHMBC and LR-HSQMBC experiments.

\section{Results and Discussion}

\section{1. $L R-H S Q M B C$ experiment}

If we consider the original LR-HSQMBC pulse sequence (Figure 1), the observable magnetization present at stage a for a general $\mathrm{CHH}$ ' spin system $\left({ }^{n} \mathrm{~J}_{\mathrm{CH}}\right.$, $J_{H H^{\prime}} \neq 0$ ) is identical to that present before acquisition in the LR-HSQC sequence (Equation 1)[19] (the effect of the G-BIRD ${ }^{r, X}$ introduced for suppressing ${ }^{1} J_{C H}$ correlations can be neglected, as it only insignificantly extends $\left(\tau=1 /{ }^{1} \mathrm{~J}_{\mathrm{CH}}\right)$ the evolution delay $\Delta$ adjusted for long-range coupling evolution). The subscript $r$ will be used subsequently throughout the whole manuscript to emphasize that the proton $\mathrm{H}^{\mathrm{r}}$ is long-range coupled to the carbon. Note that same expressions for the stepwise 
evolution of the initial $H_{z}$ magnetization in the course of the experiment will result whether the carbon is additionally coupled or not to the second proton $H^{\prime}$, i.e. whether ${ }^{n} J_{\mathrm{CH}^{\prime}} \neq 0$ or ${ }^{n} J_{\mathrm{CH}^{\prime}}=0$. The reason is that both spins $\mathrm{C}$ and $\mathrm{H}^{\prime}$ are present as $z$-magnetization during $\Delta$, and therefore the J-coupling between them remains inactive.

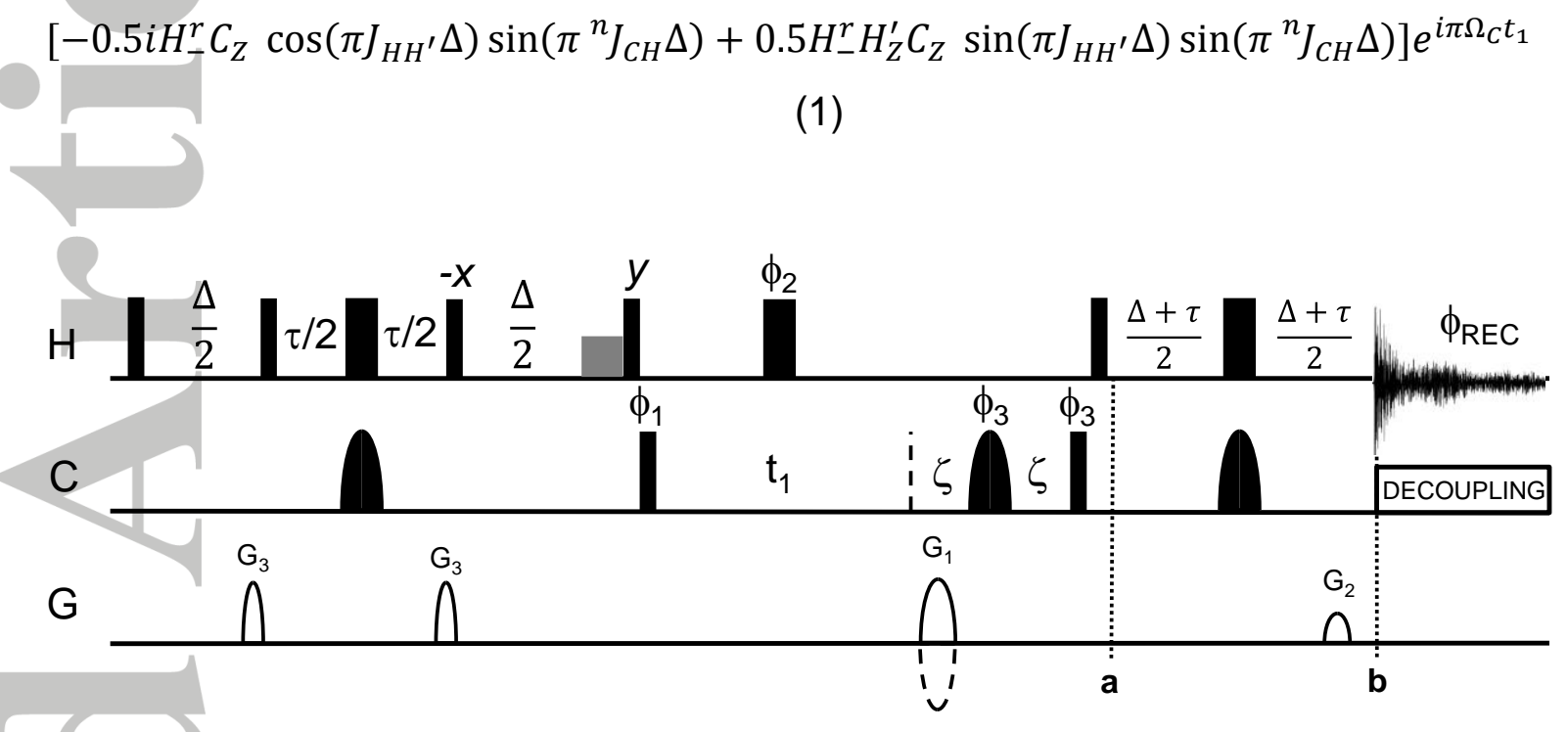

Figure 1. LR-HSQMBC pulse sequence with echo-antiecho gradient selection. [6] Thin bars represent $90^{\circ}$ pulses, thick bars $180^{\circ}$ pulses. All ${ }^{13} \mathrm{C} 180^{\circ}$ pulses are adiabatic CHIRP pulses ${ }^{[23]}$ for broadband inversion and refocusing, shown as sine shapes. A G-BIRD ${ }^{r, X}$ segment efficiently suppresses ${ }^{1} \mathrm{~J}_{\mathrm{CH}}$ responses. ${ }^{[24]} \Delta$ is the longrange coupling evolution delay and is set to an average value $0.5 /{ }^{n} J_{\mathrm{CH}}$ av and the delay $\tau$ is optimized to an average value $1 /{ }^{1} \mathrm{~J}_{\mathrm{CHav}}$. A spin lock ( $\left.1 \mathrm{~ms}\right)$, shown as a grey box, is applied before the first pair of $90^{\circ}$ pulses to suppress unwanted magnetization. Delay $\zeta$ is set to guarantee proper ${ }^{13} \mathrm{C}$ chemical shift refocusing and is equal to the length of $\mathrm{G}_{1}+$ delay for gradient recovery. The following phase cycling is applied: $\phi_{1}=x,-x ; \phi_{2}=x, x,-x,-x ; \phi_{3}=4 x, 4(-x)$; $\phi_{\text {rec }}=x,-x, x,-x,-x, x,-x, x$. Phases not shown are applied along the $x$-axis. Gradient ratios: $\mathrm{G}_{1}: \mathrm{G}_{2}: \mathrm{G}_{3}=80: 20: 34$ (odd), $-80: 20: 34$ (even). The labels $a-b$ denote the two steps of interest in the pulse sequence.

At b, after the refocusing period of length $\Delta$, the antiphase terms $i H^{r}-C_{z}$ and $H^{r}$. $H_{z}^{\prime} C_{z}$ are converted back partially into pure proton magnetization $H^{r}$. and $H^{r}-H^{\prime} z$. Note that the application of ${ }^{13} \mathrm{C}$ decoupling during acquisition cancels the antiphase terms $H^{r} C_{Z}$ and $H^{r} \cdot H_{Z}^{\prime} C_{Z}$ that are created. The full density matrix evaluation is provided in the Supplementary Material. 
$-0.5 i H_{-}^{r} C_{Z} \cos \left(\pi J_{H H^{\prime}} \Delta\right) \sin \left(\pi^{n} J_{C H} \Delta\right)+0.5 H_{-}^{r} H_{Z}^{\prime} C_{Z} \sin \left(\pi J_{H H^{\prime}} \Delta\right) \sin \left(\pi^{n} J_{C H} \Delta\right) \stackrel{\pi^{n} J_{C H} \Delta 2 H_{Z} C_{Z}}{\longrightarrow}$

\section{$\stackrel{\pi J_{H H^{\prime}} \Delta 2 H_{Z} H_{Z}^{\prime}}{\longrightarrow}$}

$$
-0.5 H_{-}^{r} \sin ^{2}\left(\pi^{n} J_{C H} \Delta\right) \cos ^{2}\left(\pi J_{H H^{\prime}} \Delta\right)-0.5 i H_{-}^{r} H_{Z}^{\prime} \sin ^{2}\left(\pi^{n} J_{C H} \Delta\right) \sin \left(2 \pi J_{H H^{\prime}} \Delta\right)
$$

Taking ${ }^{13} \mathrm{C}$ shift evolution in $\mathrm{t} 1$ into account this provides finally

$$
\left[-0.5 H{ }_{-}^{r} \sin ^{2}\left(\pi^{n} J_{C H} \Delta\right) \cos ^{2}\left(\pi J_{H H^{\prime}} \Delta\right)-0.5 i H_{-}^{r} H_{Z}^{\prime} \sin ^{2}\left(\pi^{n} J_{C H} \Delta\right) \sin \left(2 \pi J_{H H^{\prime}} \Delta\right)\right] e^{i \pi \Omega_{C} t_{1}}
$$

Interestingly, the LR-HSQMBC experiment leads to a superposition of inphase $\left(H^{r}\right.$.) and anti-phase $\left(H^{r} \cdot H^{\prime} z\right)$ multiplets with respect to the homonuclear coupling $\mathrm{JHH}$ ' respectively. Note that both terms are $90^{\circ}$ phase shifted to each other. The superposition of both terms, using the classical processing for HSQC-type data, produces final signals with the superposition of absorptive and dispersive lineshapes, noticeable in LR-HSQMBC spectra published in the literature. ${ }^{[6,7,25]}$

\section{2. $D-H M B C$}

The D-HMBC experiment (Figure 2) was introduced more than two decades ago by Furihata and Seto. ${ }^{[13]}$ Compared to the classical HMBC sequence the D$\mathrm{HMBC}$ is a refocused experiment that creates in-phase multiplets with respect to ${ }^{n} J_{C H}$ couplings allowing the application of ${ }^{13} \mathrm{C}$ broadband decoupling during the acquisition time. ${ }^{[13]}$ 


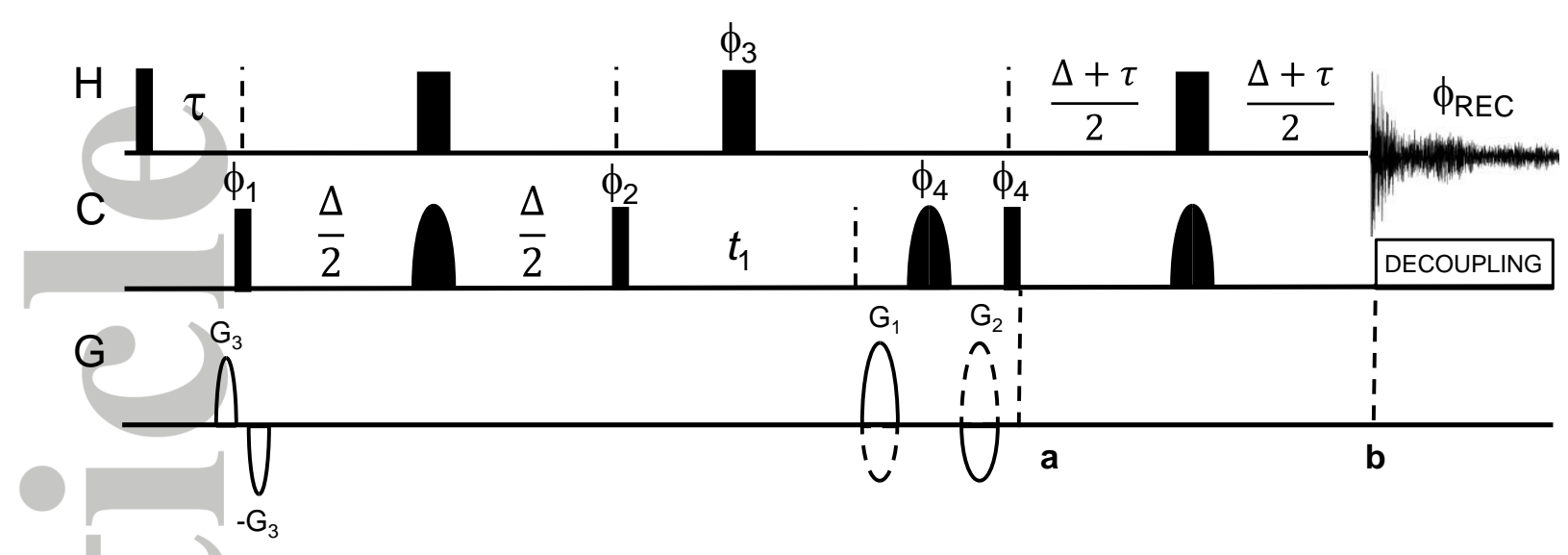

Figure 2. Pulse sequence of the slightly modified D-HMBC experiment with a pair of $180^{\circ}$ pules during the ${ }^{n} J_{\mathrm{CH}}$ evolution periods $\Delta$, gradient selection ${ }^{[26,27]}$ and with an initial low-pass $J$ filter. ${ }^{[28]}$ Thin bars represent $90^{\circ}$ pulses, thick bars $180^{\circ}$ pulses. All ${ }^{13} \mathrm{C} 180^{\circ}$ pulses are adiabatic CHIRP pulses ${ }^{[23]}$ for broadband inversion and refocusing, shown as sine shapes. $\Delta$ is the long-range coupling evolution delay and is set to an average value $0.5 /{ }^{n} J_{\text {CHAv. }} \tau$ is the one-bond coupling evolution delay and is set to an average value $0.5 /{ }^{1} \mathrm{JCHAv}$. The following phase cycling is applied: $\phi 1=x$, $x,-x,-x ; \phi 2=x,-x ; \phi 3=4 x, 4(-x) ; \phi 4=8 x, 8(-x) ; \phi r e c=4(x,-x), 4(-x, x)$. Pulses with no phase indicated are applied along the $x$-axis. Gradient ratios: $\mathrm{G}_{1}: \mathrm{G}_{2}: \mathrm{G}_{3}=50:-30: 34$ (odd), $-30: 50: 34$ (even). The labels $a-b$ denote the two stages of interest in the pulse sequence.

The observable magnetization present at stage a for a general $\mathrm{CHH}^{\prime}$ spin system is identical to that present before acquisition in the classical HMBC sequence ${ }^{[19]}$ (the effect of the low-pass $J$ filter for suppressing ${ }^{1} J_{\mathrm{CH}}$ correlations can be neglected, as it only marginally reduces the intensity of the long-range coupling magnetization):[14, 28]

$$
\left[0.5 H_{-}^{r} C_{Z} \cos \left(\pi J_{H H^{\prime}} \Delta\right) \sin \left(\pi^{n} J_{C H} \Delta\right)+i H_{-}^{r} H_{Z}^{\prime} C_{Z} \sin \left(\pi J_{H H^{\prime}} \Delta\right) \sin \left(\pi^{n} J_{C H} \Delta\right)\right] e^{i \pi \Omega_{C} t_{1}}
$$

During the refocusing period of length $\Delta$, the antiphase terms $\mathrm{H}^{r}-\mathrm{CZ}_{z}$ and $i \mathrm{H}^{r}$. $H^{\prime} z C z$ are converted back partially into pure proton magnetization $H^{r}$. and $H^{r} \cdot H^{\prime} z$. Note that the application of ${ }^{13} \mathrm{C}$ decoupling during acquisition cancels the antiphase terms $H^{r}-C_{z}$ and $H^{r}-H^{\prime} z C_{z}$ as well. The full density matrix evaluation is provided in the Supplementary Material. 
$0.5 H_{-}^{r} C_{Z} \cos \left(\pi J_{H H^{\prime}} \Delta\right) \sin \left(\pi^{n} J_{C H} \Delta\right)+i H_{-}^{r} H_{Z}^{\prime} C_{Z} \sin \left(\pi J_{H H^{\prime}} \Delta\right) \sin \left(\pi^{n} J_{C H} \Delta\right) \stackrel{\pi^{n} J_{C H} \Delta 2 H_{Z} C_{Z}}{\longrightarrow}$

$$
\stackrel{\pi J_{H H^{\prime}} \Delta 2 H_{Z} H_{Z}^{\prime}}{\longrightarrow}
$$

$0.5 i H_{-}^{r} C_{Z} \sin ^{2}\left(\pi^{n} J_{C H} \Delta\right) \cos \left(2 \pi J_{H H^{\prime}} \Delta\right)-H_{-}^{r} H_{Z}^{\prime} \sin ^{2}\left(\pi^{n} J_{C H} \Delta\right) \sin \left(2 \pi J_{H H^{\prime}} \Delta\right)$

Taking ${ }^{13} \mathrm{C}$ shift evolution in $\mathrm{t} 1$ into account this provides finally

$\left[0.5 i H_{-}^{r} \sin ^{2}\left(\pi^{n} J_{C H} \Delta\right) \cos \left(2 \pi J_{H H^{\prime}} \Delta\right)-H_{-}^{r} H_{Z}^{\prime} \sin ^{2}\left(\pi^{n} J_{C H} \Delta\right) \sin \left(2 \pi J_{H H^{\prime}} \Delta\right)\right] e^{i \pi \Omega_{C} t_{1}}$

It follows that also cross peaks in D-HMBC spectra result from a superposition of in-phase $\left(H^{r}-\right)$ and anti-phase $\left(H^{r} \cdot H^{\prime} z\right)$ multiplets with respect to the homonuclear coupling $\mathrm{JHH}$. Note that both terms are $90^{\circ}$ phase shifted to each other as well, but (as a whole) also $90^{\circ}$ out of phase compared to the response of the LR-HSQMBC experiment. The superposition of both terms, using the classical processing for HSQC-type data, produces final signals with the superposition of absorptive and dispersive lineshapes. Yet, D-HMBC spectra published in the literature so far were displayed in magnitude mode. ${ }^{[13,14]}$

\subsection{Comparison of the refocused experiments $L R-H S Q M B C$ and D-HMBC}

To analyze the performance and the characteristics of the refocused LR-HSQMBC and the D-HMBC experiments, the observable coherences present before data acquisition are listed below (Table 1). For comparison, the corresponding coherences found for the non-refocused variants LR-HSQC and HMBC have been included. [19] 


\begin{tabular}{|c|c|}
\hline LR-HSQMBC (refocused) & $\begin{array}{l}{\left[-0.5 H_{-}^{r} \sin ^{2}\left(\pi^{n} J_{C H} \Delta\right) \cos ^{2}\left(\pi J_{H H^{\prime}} \Delta\right)\right]} \\
\left.-0.5 i H_{-}^{r} H_{Z}^{\prime} \sin ^{2}\left(\pi^{n} J_{C H} \Delta\right) \sin \left(2 \pi J_{H H^{\prime}} \Delta\right)\right] e^{i \pi \Omega_{C} t_{1}}\end{array}$ \\
\hline -HMBC (refocused) & $\begin{array}{l}{\left[0.5 i H_{-}^{r} \sin ^{2}\left(\pi^{n} J_{C H} \Delta\right) \cos \left(2 \pi J_{H H^{\prime}} \Delta\right)\right.} \\
\left.-H_{-}^{r} H_{Z}^{\prime} \sin ^{2}\left(\pi^{n} J_{C H} \Delta\right) \sin \left(2 \pi J_{H H^{\prime}} \Delta\right)\right] e^{i \pi \Omega_{C} t_{1}}\end{array}$ \\
\hline LR-HSQC (non-refocused) & $\begin{array}{l}{\left[-0.5 i H_{-}^{r} C_{Z} \sin \left(\pi^{n} J_{C H} \Delta\right) \cos \left(\pi J_{H H^{\prime}} \Delta\right)\right.} \\
\left.+0.5 H_{-}^{r} H_{Z}^{\prime} C_{Z} \sin \left(\pi^{n} J_{C H} \Delta\right) \sin \left(\pi J_{H H^{\prime}} \Delta\right)\right] e^{i \pi \Omega_{C} t_{1}}\end{array}$ \\
\hline HMBC (non-refocused) & $\begin{array}{l}{\left[0.5 H_{-}^{r} C_{Z} \sin \left(\pi^{n} J_{C H} \Delta\right) \cos \left(\pi J_{H H^{\prime}} \Delta\right)\right.} \\
\left.+i H_{-}^{r} C_{Z} H_{Z}^{\prime} \sin \left(\pi^{n} J_{C H} \Delta\right) \sin \left(\pi J_{H H^{\prime}} \Delta\right)\right] e^{i \pi \Omega_{C} t_{1}}\end{array}$ \\
\hline
\end{tabular}

Table 1. Product operator expressions for the observable coherences present before data acquisition for the refocused LR-HSQMBC and D-HMBC and the non-refocused LR-HSQC and the HMBC experiments. For each experiment, the final detected signal is a superposition of two different coherences.

The following conclusions can be drawn from the product operator expressions for the observable coherences:

- For any of the four experiments the final signal is a superposition of two types of multiplets with different numerical factors: an in-phase $\left(H^{r}-\right)$ and an antiphase $\left(H^{r} \cdot H_{Z}^{\prime}\right)$ multiplet with respect to $J_{H H^{\prime}}$ for the refocused LR-HSQMBC and D-HMBC experiments and an anti-phase $\left(\mathrm{H}^{r}-\mathrm{C}_{Z}\right)$ and a doubly anti-phase $\left(H^{\prime}-H^{\prime}{ }_{Z} C_{Z}\right)$ multiplet with respect to $J_{C H}$ and $J_{H H}$ respectively for the nonrefocused LR-HSQC and HMBC experiments. In addition, different numerical factors are involved, i.e. the two superimposed multiplets contribute equally for LR-HSQMBC (refocused and non-refocused) and differently for D-HMBC (refocused and non-refocused). 
- The four experiments are characterized by different trigonometric expressions for the corresponding two superimposed multiplets:

When varying the ${ }^{n} J_{\mathrm{CH}}$-evolution delay $\Delta$ the cross-peak intensities are affected by ${ }^{n} J_{C H}$ as expected, i.e. with a $\sin ^{2}\left(\pi^{n} J_{C H} \Delta\right.$ )-dependence (LRHSQMBC and D-HMBC) and $\sin \left(\pi^{n} J_{C H} \Delta\right)$ - dependence (LR-HSQC and $\mathrm{HMBC})$.

- With respect to $\mathrm{JHH}$ on the other hand, the cross-peak intensities are affected differently if we compare the refocused and non-refocused experiments. $A \sin \left(\pi^{n} J_{H H^{\prime}} \Delta\right)$ - and $\cos \left(\pi^{n} J_{H H^{\prime}} \Delta\right)$-dependence is observed for the anti-phase terms $H^{r} \cdot H^{\prime} z$ and $H^{r} \cdot H^{\prime} z C_{z}$ in both LR-HSQC and HMBC, respectively. However, a $\sin \left(2 \pi^{n} J_{H H^{\prime}} \Delta\right)$-dependence is observed for the anti-phase term $H^{r} \cdot H^{\prime} z$ in both LR-HSQMBC and D-HMBC, but a $\cos ^{2}\left(\pi^{n} J_{H H^{\prime}} \Delta\right)$ dependence is observed for the in-phase term $H^{r}$. in the LRHSQMBC experiment, and a $\cos \left(2 \pi^{n} J_{H H^{\prime}} \Delta\right)$ dependence is observed for the in-phase term $H^{r}$ - in the D-HMBC experiment.

It turns out that due to the different trigonometric factors found in the four experiments, different sensitivity dependence as a function of $\Delta$ are expected and have been investigated.

The ${ }^{n} J_{\mathrm{CH}}$-influence on the $\Delta$-dependence of cross-peak intensities, though different for the refocused and non-refocused experiments, is well-known. Note that with non-ideally adjusted delays $\Delta$ the intensities of the individual multiplet lines will additionally decrease with the refocused experiments compared to the nonrefocused counterparts because of the $\sin ^{2}\left(\pi^{n} J_{C H} \Delta\right)$-rather the $\sin \left(\pi^{n} J_{C H} \Delta\right)$-dependencies respectively. However, at least part (if not more) of this sensitivity loss is compensated by the partial signal collapse achieved with ${ }^{13} \mathrm{C}$ broadband decoupling.

Therefore, our investigation is focused on the effect of the numerical factors valid for the two multiplet components and on the influence of the trigonometric $\mathrm{JHH}^{\prime}$ factors, particularly for the refocused LR-HSQMBC and D-HMBC experiment on their sensitivity.

The equations 2 and 4 show that the in-phase $\left(H^{r}-\right)$ multiplets have a numerical coefficient that is identical in both experiments, while the coefficients of the 
anti-phase $\left(H^{r}-H^{\prime} z\right)$ multiplets have a numerical coefficient that is two times as large for D-HMBC compared to LR-HSQMBC. The same behavior applies to their nonrefocused experiment counterparts. ${ }^{[19]}$

Concentrating now on the refocused experiments and since the effect of the trigonometric functions of equations $2 \& 4$ are "visually" difficult to estimate, we have evaluated numerically - taking into account the different numerical factors but omitting the $\sin ^{2}\left(\pi J_{C H} \Delta\right)$ factor - both in-phase $\left(H_{-}^{r}\right)$ and anti-phase $\left(H^{r}-H_{Z}^{\prime}\right)$ terms for four $\mathrm{JHH}^{\prime}$ specific values of $\Delta$ for the D-HMBC and LR-HSQMBC experiments, respectively (Table 2 ).

\begin{tabular}{|c|c|c|c|c|}
\hline . & \multicolumn{2}{|c|}{ LR-HSQMBC } & \multicolumn{2}{|c|}{ D-HMBC } \\
\hline$\Delta$ & $\begin{array}{c}H^{r} \\
-0.5 \cos ^{2}\left(\pi J_{H H^{\prime} \Delta}\right)\end{array}$ & $\begin{array}{c}H^{r} \cdot H^{\prime} z \\
-0.5 i \sin \left(2 \pi J_{H H^{\prime}} \Delta\right)\end{array}$ & $\begin{array}{c}H^{r} . \\
0.5 i \cos \left(2 \pi J_{H H^{\prime} \Delta}\right)\end{array}$ & $\begin{array}{c}H^{r} \cdot H^{\prime} z \\
-\sin \left(2 \pi J_{\left.H H^{\prime} \Delta\right)}\right.\end{array}$ \\
\hline $0.25 / J_{H H^{\prime}}$ & -0.25 & -0.5 & 0 & -1 \\
\hline $0.5 / J_{\mathrm{HH}^{\prime}}$ & 0 & 0 & -0.5 & 0 \\
\hline $0.75 / J_{\mathrm{HH}}$ & -0.25 & 0.5 & 0 & +1 \\
\hline $1 / J_{\mathrm{H}^{\prime}}$ & -0.5 & 0 & +0.5 & 0 \\
\hline
\end{tabular}

Table 2. Numerical evaluation of both in-phase $\left(H_{-}\right)$and anti-phase ( $\left.H_{-} H_{z}^{\prime}\right)$ terms present before acquisition in D-HMBC and LR-HSQMBC experiments, for four different values of $\Delta$. The trigonometric factor $\sin ^{2}\left(\pi J_{C H} \Delta\right)$ is assumed to be equal to 1.

From this numerical evaluation it is quite obvious that with respect to $\mathrm{JHH}_{\mathrm{H}}$ the two experiments behave differently:

(i) In the absence of homonuclear couplings, i.e. with $J_{H H^{\prime}}=0$, due to the same $\sin ^{2}\left(\pi J_{\mathrm{CH}} \Delta\right)$-dependence with zeroes at $\Delta=\mathrm{k}^{\mathrm{n}} \mathrm{J}_{\mathrm{CH}}$, equal intensities for corresponding cross-peaks are obtained in both spectra.

(ii) Neglecting the different signs and with the exception of $\Delta=k / J_{H H}$, more intense in-phase $\left(H_{-}^{r}\right)$ and anti-phase $\left(H_{-}^{r} H_{z}^{\prime}\right)$ components result for the D-HMBC experiment. Hence more intense cross-peaks are expected to be observed in the D-HMBC- compared to the LR-HSQMBC-spectrum.

(iii) Additional intensity zeroes occur exclusively for the LR-HSQMBC experiment at $\Delta=(0.5+k) / J_{H H}$. This is due to the different $J_{H H^{\prime}}$-dependence of the in-phase 
and anti-phase components with the two experiments. Whereas with their $\cos \left(2 \pi J_{H} H^{\prime} \Delta\right)$ - and $\sin \left(2 \pi J_{H H^{\prime}} \Delta\right)$-dependence the two components counterbalance each other and at least one of them is $>0$ with the D-HMBC, these undesirable additional zeroes result with the refocused (but not with the non-refocused LR-HSQC) LR-HSQMBC experiment due to its $\cos ^{2}\left(\pi J_{H H^{\prime}} \Delta\right)$ and $\sin \left(2 \pi J_{H H^{\prime}} \Delta\right)$-dependence of the in-phase and anti-phase components respectively.

(iv) Pure (in-phase) $H^{r}$--multiplets will show up in both spectra for $\Delta=k / J_{H H^{\prime}}$ and in the D-HMBC-spectrum additionally for $\Delta=0.5+\mathrm{k} / \mathrm{JHH}^{\prime}$. In contrast, pure (antiphase) $H^{r}$-H'-multiplets will show up exclusively in the D-HMBC spectra with $\Delta=$ $(0.25+0.5 k) / \mathrm{JH}^{\prime}$.

(v) This peculiarity of the D-HMBC is not that important in routine experiments but may become problematic with ${ }^{n} J_{\mathrm{CH}}$-evolution delay $\Delta$ adjusted for small ${ }^{n} J_{\mathrm{CH}}$ and with small $J_{H H}$ couplings. Under these circumstances, $\Delta$ may be close or equal to $\Delta=(0.25+0.5 \mathrm{k}) / \mathrm{JHH}^{\prime}$, hence allowing the anti-phase $H^{r} \cdot H^{\prime} z$ term to become dominant. However, multiplet lines in anti-phase may cancel each other, partially or completely in the worst case.

This problem has already been recognized and addressed with small heteronuclear long-range couplings ${ }^{[6]}$, but obviously arises also with small homonuclear couplings in combination with long delays $\Delta$. It occurs also, but because of the non-vanishing $\mathrm{H}^{r}$ - term - by far less pronounced with the LRHSQMBC experiment.

This inherent problem of the D-HMBC with small $\mathrm{JHH}^{\prime}$-coupling constants is exacerbated with increased line broadening and with poor digital resolution in the F2 dimension and in case of additional (small) proton-proton couplings. Note that since both LR-HSQMBC and D-HMBC utilize ${ }^{13} \mathrm{C}$ decoupling, the digital resolution in F2 is inherently limited, as the acquisition time must be typically kept $<500 \mathrm{~ms}$ for preserving the probeheads from damages. The problem of cancelation of antiphase terms is not limited to $\Delta=(0.25+0.5 \mathrm{k}) / \mathrm{JHH}^{\prime}$ but is also noticeable for small couplings $(\mathrm{J}<3 \mathrm{~Hz})$ and long delays $\Delta$ in general. For small scalar coupling constants, inphase (IP) cross-peaks are always larger than antiphase (AP) and the IP/AP ratio grow to $>3$ as the coupling constants values are below $3 \mathrm{~Hz}$ and to $>10$ for values 
below $1.5 \mathrm{~Hz}$ if Lorentzian line shapes with line width at half-height of $7 \mathrm{~Hz}$ are assumed. ${ }^{[6]}$

Based on these findings we derived some rules for an optimal use of the $D$ HMBC and LR-HSQMBC experiments:

(i) If $J_{H H}$ is large $(\mathrm{J}>5 \mathrm{~Hz})$ their multiplet lines are well-resolved and both components $H^{r}$ - and $H^{r}-H^{\prime} z$ contribute to the cross peak's intensity, irrespective of the length of $\Delta$. If $H^{r}-H^{\prime} z$ dominates no mutual cancellation of the well-spaced anti-phase multiplet lines will occur. However, in contrast to D-HMBC spectra the cross-peaks in LR-HSQMBC spectra may become small or even vanish close or equal to $\Delta=k+0.5 / J_{\mathrm{HH}}$ respectively.

D-HMBC is clearly superior to LR-HSQMBC.

Compare Figs. 3 and 4 , in particular and with $\mathrm{JHH}^{\prime}=10 \mathrm{~Hz}$ the cross-peaks intensities for the two experiments at $\Delta=50$ and $150 \mathrm{~ms}$.

(ii) If $J_{H H^{\prime}}$ is small (e.g. $1-3 \mathrm{~Hz}$ ) again both components $H^{r}$. and $H^{r}$. $H_{Z}^{\prime}$ theoretically contribute to the cross peak's intensity. However, due to partial or complete mutual cancellation of the closely spaced anti-phase signals of the $H^{r}-H_{z}^{\prime}$ multiplet with small $J_{H H^{\prime}}$, mainly (in the worst case exclusively) the in-phase $H^{r}$. multiplet contributes and determines the cross peak's intensity. This effect, valid for both experiments, does not manifest for very small ${ }^{n} J_{\mathrm{CH}}$-evolution delays $\Delta$, for which the in-phase component $H^{r}$ - already dominates. However, with increasing $\Delta$ delays this "small- $J_{H H}$-effect" can no longer be neglected and affects the performance of the D-HMBC- and the LR-HSQMBC-experiment differently: According to Table 2, in D-HMBC spectra, an attenuation of cross peaks due to this effect is expected to appear for ${ }^{n} J_{C H}$-delays close/equal to $\Delta=$ $(0.5 k+0.25) / J_{H H}, k=0,1,2, \ldots, n$, where the anti-phase $H^{r} \cdot H_{Z}^{\prime}$ component dominates. For $\mathrm{JHH}^{\prime}=1.5 \mathrm{~Hz}$, the first "weak" or "zero" intensity point caused by this effect occurs for $\Delta=167 \mathrm{~ms}$. With the LR-HSQMBC experiment this same peculiarity with small $\mathrm{JHH}_{\mathrm{H}}$ exists as well and is expected to show up at values to $\Delta=(k+0.5) / J_{H H}, k=0,1,2, \ldots$, where the anti-phase $H^{r} H_{z}^{\prime}$ component dominates. For $\mathrm{JHH}{ }^{\prime}=1.5 \mathrm{~Hz}$, the first "weak" or "zero" intensity point caused by this effect occurs for $\Delta=333 \mathrm{~ms}$. However, at these $\Delta$ values the intensity of both components is already close to zero, irrespective of the size of $\mathrm{JHH}_{\mathrm{H}}$. Hence 
only an insignificant additional decrease in sensitivity has to be taken into account. Furthermore this inconveniency, i.e. the first "zero" arises at a ${ }^{n} \mathrm{JCH}^{-}$ delay twice as large compared to the D-HMBC, well above the typical range used with these experiments. Consequently, this problem with small $J_{H H}$ is less manifest with LR-HSQMBC.

LR-HSQMBC is equal or superior to D-HMBC.

Compare in Fig. 5 the cross-peaks intensities for the two experiments at $\Delta=50$ and $150 \mathrm{~ms}$ with $J_{\mathrm{HH}}=4.5$ and $1.5 \mathrm{~Hz}$.

\subsection{Simulation results}

To corroborate the analytical findings and the corresponding conclusions a few numerical simulations taking advantage of the BRUKER NMRSIM program have been performed. These simulations also serve to study unforeseeable effects arising from the overlap of the different lineshapes (e.g. absorptive in-phase and dispersive anti-phase) of the two components $H^{r}$ - and $H^{r}-H^{\prime} z$ and to investigate more extended spin-systems with additional homonuclear couplings. The behavior of the two experiments will be studied in a wide $\Delta$-range encompassing experimental setups adjusted for large (e.g. $10 \mathrm{~Hz}$ ) and very small (e.g. $1.5 \mathrm{~Hz})^{n} \mathrm{~J}_{\mathrm{CH}}$-couplings with typical $\Delta$-delays of $50 \mathrm{~ms}$ and $200 \mathrm{~ms}$ respectively. Two situations with $\mathrm{JHH}^{-}$-values $>5 \mathrm{~Hz}$ and $<5 \mathrm{~Hz}$ will be examined separately.

\subsubsection{Homonuclear couplings $\mathrm{J}_{\mathrm{HH}}>5 \mathrm{~Hz}$}

In Figure 3 and 4 , simulated spectra obtained for a $\mathrm{CH}^{r} \mathrm{H}^{\prime}$ spin system $\left({ }^{n} \mathrm{~J}_{\mathrm{CH}}=4\right.$ $\mathrm{Hz}, \mathrm{JHH}^{\prime}=10 \mathrm{~Hz}, \mathrm{~T}_{2}=0.4 \mathrm{~s}$ for all nuclei) using the LR-HSQMBC and the D-HMBC pulse sequences are shown. 


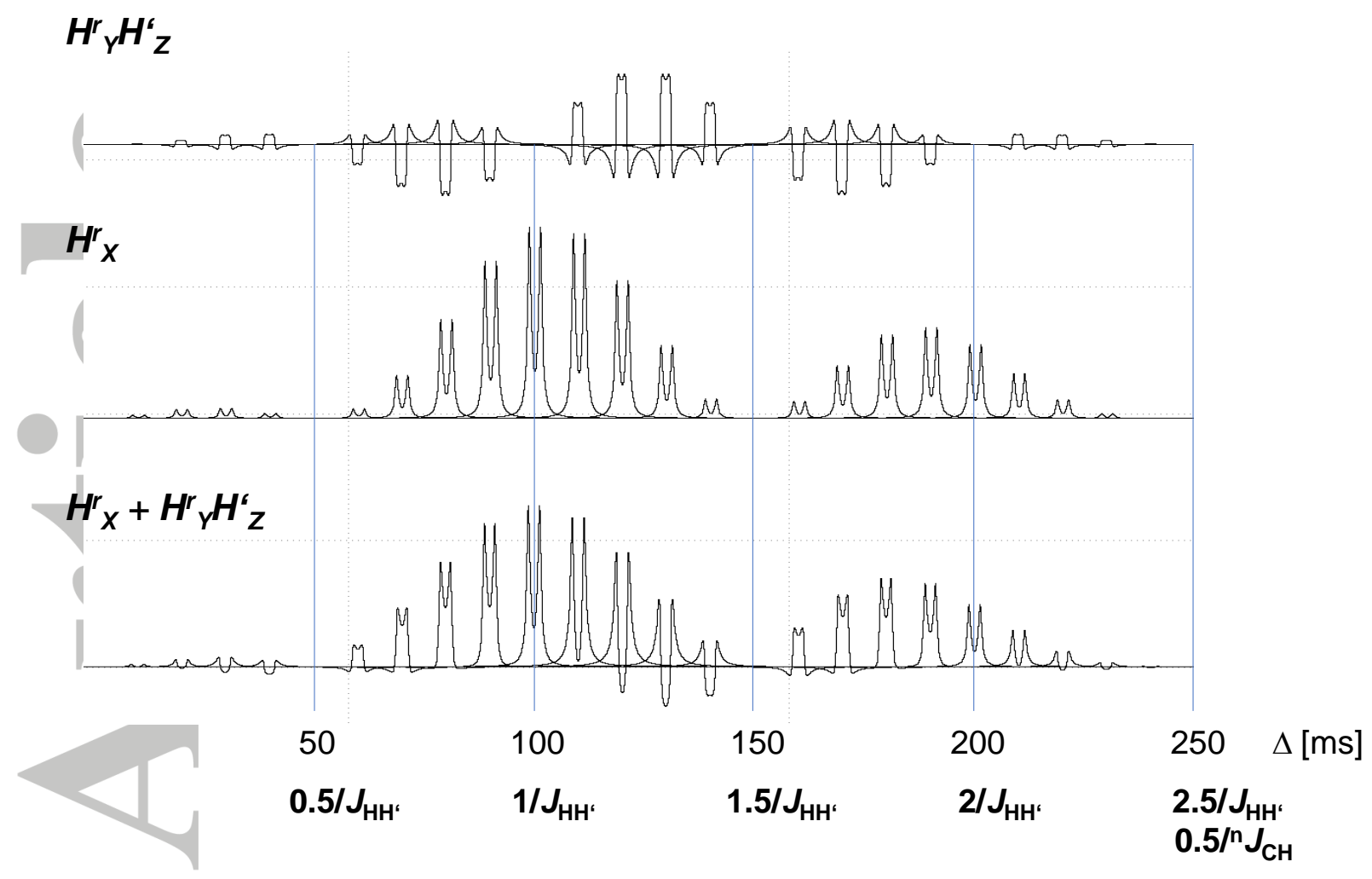

Figure 3. Simulated spectra obtained for a $\mathrm{CH}^{r} \mathrm{H}^{\prime}$ spin system using the LRHSQMBC pulse sequence showing the final signal detected (bottom), only the inphase $\boldsymbol{H}^{r}{ }_{x}$ component (middle), and only the anti-phase component $\boldsymbol{H}^{r} \boldsymbol{H}^{\prime} \boldsymbol{z}$ (top). For the separate measurement of both terms $\boldsymbol{H}^{r} \boldsymbol{x}$ and $\boldsymbol{H}^{r} \boldsymbol{H}^{\prime}{ }_{z}$, respectively, two experiments were measured separately. $\operatorname{Exp}(1)$ : before acquisition, an additional $90^{\circ} x^{-1} H$ pulse was added: $\rightarrow$ only $\boldsymbol{H}^{r} x$ will be detected. $\operatorname{Exp}(2)$ : standard LRHSQMBC experiment, with the detection of both $\boldsymbol{H}^{r} \boldsymbol{x}$ and $\boldsymbol{H}^{r} \boldsymbol{y} \boldsymbol{H}^{\prime} \mathbf{z}$ components. $\boldsymbol{H}^{r} \boldsymbol{H}^{\prime} \mathbf{z}$ is obtained by subtracting both experiments. The delay $\Delta$ was varied from 0 to 250 ms in steps of $10 \mathrm{~ms}$. Parameters used: ${ }^{\mathrm{n}} \mathrm{J}_{\mathrm{CH}}=4 \mathrm{~Hz}, J_{\mathrm{HH}}=10 \mathrm{~Hz}$. The relaxation time $T_{2}$ has been set to $0.4 \mathrm{~s}$ for all nuclei and relaxation during the whole sequence was taken into account. The spectra are displayed at the same signal-to-noise level. Simulations have been performed with the BRUKER NMRSIM program for WINDOWS (version 5.5.3. 2012). 


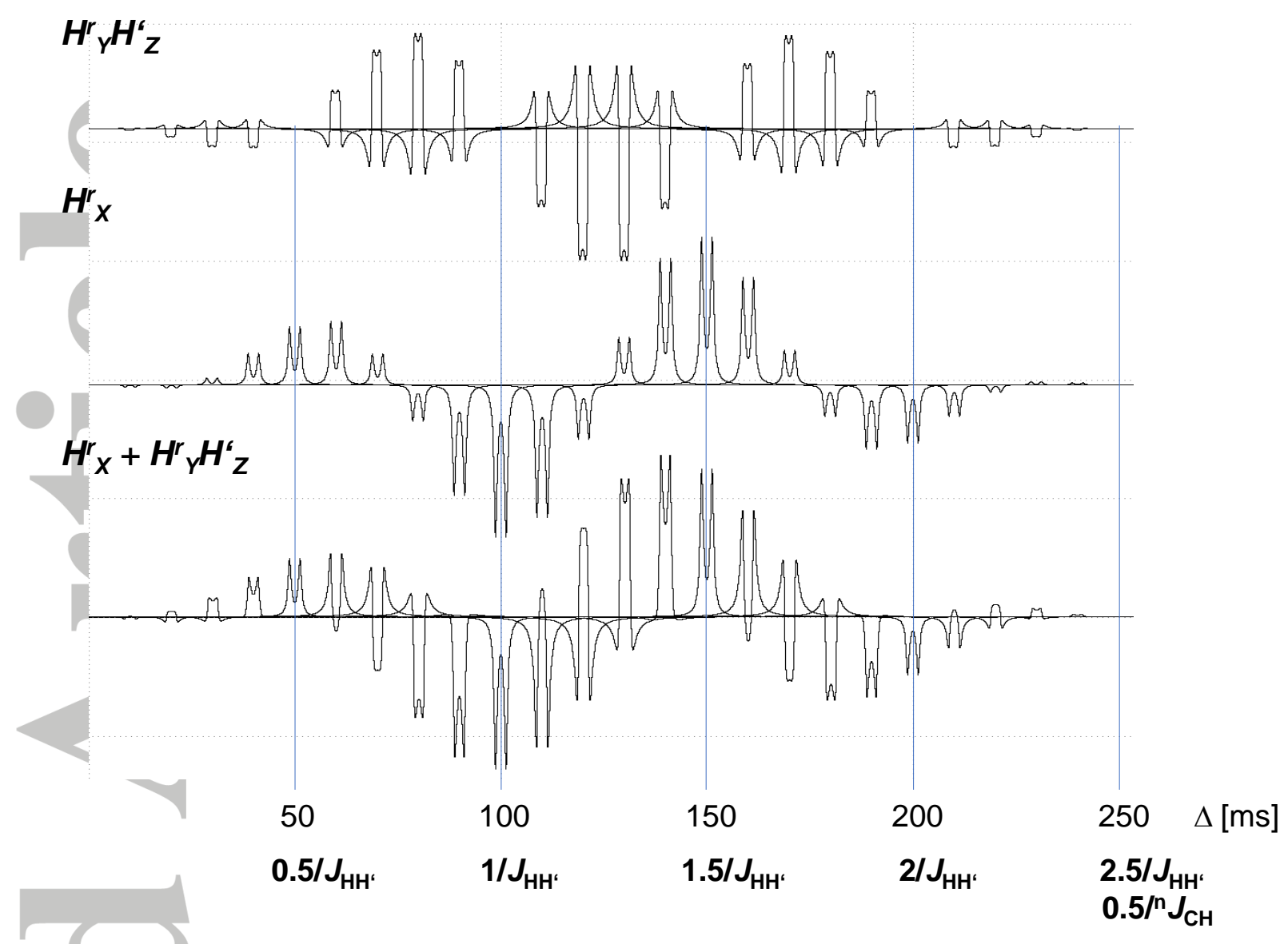

Figure 4. Simulated spectra obtained for a $\mathrm{CH}^{\prime} \mathrm{H}^{\prime}$ spin system using the D-HMBC pulse sequence showing the final signal detected (bottom), only the in-phase $\boldsymbol{H}^{r} \boldsymbol{x}$ component (middle), and only the anti-phase component $\boldsymbol{H}^{r} \boldsymbol{H}^{\prime} \boldsymbol{z}$ (top). For the separate measurement of both terms $\boldsymbol{H}^{r} \boldsymbol{x}$ and $\boldsymbol{H}^{r} \boldsymbol{H}^{\prime}$ 'z, respectively, two experiments were measured separately. $\operatorname{Exp}(1)$ : before acquisition, an additional $90^{\circ} x^{-1} \mathrm{H}$ pulse

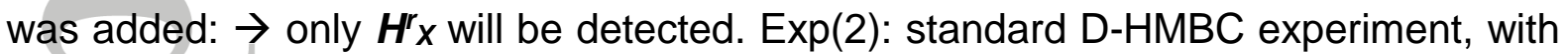
the detection of both $\boldsymbol{H}^{r} \boldsymbol{x}$ and $\boldsymbol{H}^{r} \boldsymbol{H}^{\prime} \boldsymbol{z}$ components. $\boldsymbol{H}^{r} \boldsymbol{H}^{\prime} \boldsymbol{z}$ is obtained by subtracting both experiments. For clarity and for an easier comparison with the LR-HSQMBC experiment, an additional $90^{\circ}$ phase correction was added, such as the in-phase terms in both experiments can be represented in absorption mode. The delay $\Delta$ was varied from 0 to $250 \mathrm{~ms}$ in steps of $10 \mathrm{~ms}$. Parameters used: ${ }^{\mathrm{n}} \mathrm{J}_{\mathrm{CH}}=4 \mathrm{~Hz}, \mathrm{JHH}^{\prime}=10$ $\mathrm{Hz}$. The relaxation time $\mathrm{T}_{2}$ has been set to $0.4 \mathrm{~s}$ for all nuclei and relaxation was taken into account during the whole sequence. The spectra are displayed at the same signal-to-noise level. Simulations have been performed with the BRUKER NMRSIM program for WINDOWS (version 5.5.3. 2012).

It can be seen that the intensity of the simulated resonance as a function of $\Delta$ follows a regular pattern with the LR-HSQMBC sequence, with zeroes at $\Delta=1 / \mathrm{n} J_{\mathrm{CH}}$ (250 ms) and $\Delta=(k+0.5) / J_{H H^{\prime}}(50$ and $150 \mathrm{~ms})$ respectively. On the other hand, for 
the D-HMBC, according to theory (Equations $2 \& 4$ in Table 1 and specific values in Table 2) and in agreement with the results found for the non-refocused variants, the intensities are generally higher. Most noteworthy and in contrast to LR-HSQMBC no $\mathrm{JHH}_{\mathrm{H}-\mathrm{caused}}$ zeroes arise and the unique zero in the spectra occurs for $\Delta=250 \mathrm{~ms}$, that is at $\Delta=1 /{ }^{n} J_{\mathrm{CH}}{ }^{[19]}$ Note also that in accordance with theory (Table 2) a pure inphase component $\boldsymbol{H}^{r} \boldsymbol{x}$ results for the LR-HSQMBC experiment exclusively at $\Delta=$ $k / J_{H H}$. On the other hand a pure in-phase component $\boldsymbol{H}^{r} \boldsymbol{x}$ and a pure anti-phase component $\boldsymbol{H}^{r} \boldsymbol{y} \boldsymbol{H}^{\prime} \boldsymbol{z}$ shows up for the D-HMBC experiment at $\Delta=0.5 \mathrm{k} / \mathrm{J}_{\mathrm{HH}}$ and $\Delta=$ $(0.5 \mathrm{~K}+0.25) / \mathrm{JHH}^{\prime}$ respectively. Note also that the different behavior of the two experiments is not restricted only to these zeroes and the different behavior of the two components, but the general sensitivity decrease of the LR-HSQMBC experiment compared to $\mathrm{D}-\mathrm{HMBC}$ which is most pronounced in the $\Delta$-region close to the $\mathrm{JHH}^{\prime}$ induced zeroes.

Therefore, and as long as the $\mathrm{JHH}^{\prime}$ coupling is not too small, i.e. as long as the multiplet lines are well resolved, the risk for missing correlation signals with small long-range couplings ${ }^{n} J_{C H}$ in LR-HSQMBC spectra is potentially higher than in DHMBC spectra. This is also in line with the results of Williamson et al. ${ }^{[6]}$ who found that the theoretical overall intensity is higher using the D-HMBC pulse sequence, particularly for smaller values of $\Delta$.

\subsubsection{Homonuclear couplings $\mathrm{J}_{\mathrm{H} \mathrm{H}^{\prime}}<5 \mathrm{~Hz}$}

As revealed by our theoretical evaluation, the LR-HSQMBC is potentially superior to the D-HMBC as soon as very small homonuclear couplings $\mathrm{JHH}_{\mathrm{H}}$ are present. This superiority is more pronounced with short $T_{2}$ relaxation times and most likely when several small $J_{\mathrm{HH}}$ couplings are involved. As outlined for the $\mathrm{CH}^{\mathrm{r}} \mathrm{H}^{\prime}$-spin system with a small $\mathrm{J}_{H H^{\prime}}$ coupling constant the $\boldsymbol{H}^{r}{ } \boldsymbol{H}^{\prime} \mathbf{z}$ (anti-phase) component may be weakened (or will even vanish) due to mutual cancellation of closely spaced antiphase multiplet lines in both experiments. The risk of mutual cancelation increases with decreasing $\mathrm{JHH}^{\prime}$ and starts to affect the intensity of the anti-phase component most severely with $J_{H H^{\prime}}<2 \mathrm{~Hz}$. In the $\Delta$-range 0 to $1 / J_{H H^{\prime}}$ this occurs around $\Delta=$ $0.25 / J_{H} H^{\prime}$ and $0.75 / J_{H H^{\prime}}$ with the D-HMBC and around $\Delta=0.5 / J_{H H^{\prime}}$ with the LRHSQMBC experiment. Hence with values $\Delta<0.5 / J_{H H^{\prime}}$ this peculiarity with small $J_{H H^{\prime}}$ 
couplings affects sensitivity only with the D-HMBC but not with the LR-HSQMBC experiment. Consequently, under these circumstances, the sensitivity of the LRHSQC experiment will be superior and more ${ }^{n} J_{C H}$ cross-peaks (originating from protons with small additional $\mathrm{J}_{\mathrm{HH}}$ couplings) are expected to show up compared to the D-HMBC spectra as corroborated in the literature. ${ }^{[6]}$

Corresponding simulations with a $\mathrm{CHH}$ ' spin system ( ${ }^{n} \mathrm{~J}_{\mathrm{CH}}=4 \mathrm{~Hz}, \mathrm{JHH}^{\prime}=4.5$ $\mathrm{Hz}$ or $J_{H H^{\prime}}=1.5 \mathrm{~Hz}, \mathrm{~T}_{2}=0.4 \mathrm{~s}$ for all nuclei) using the LR-HSQMBC and the D-HMBC pulse sequences are shown in Figure 5.
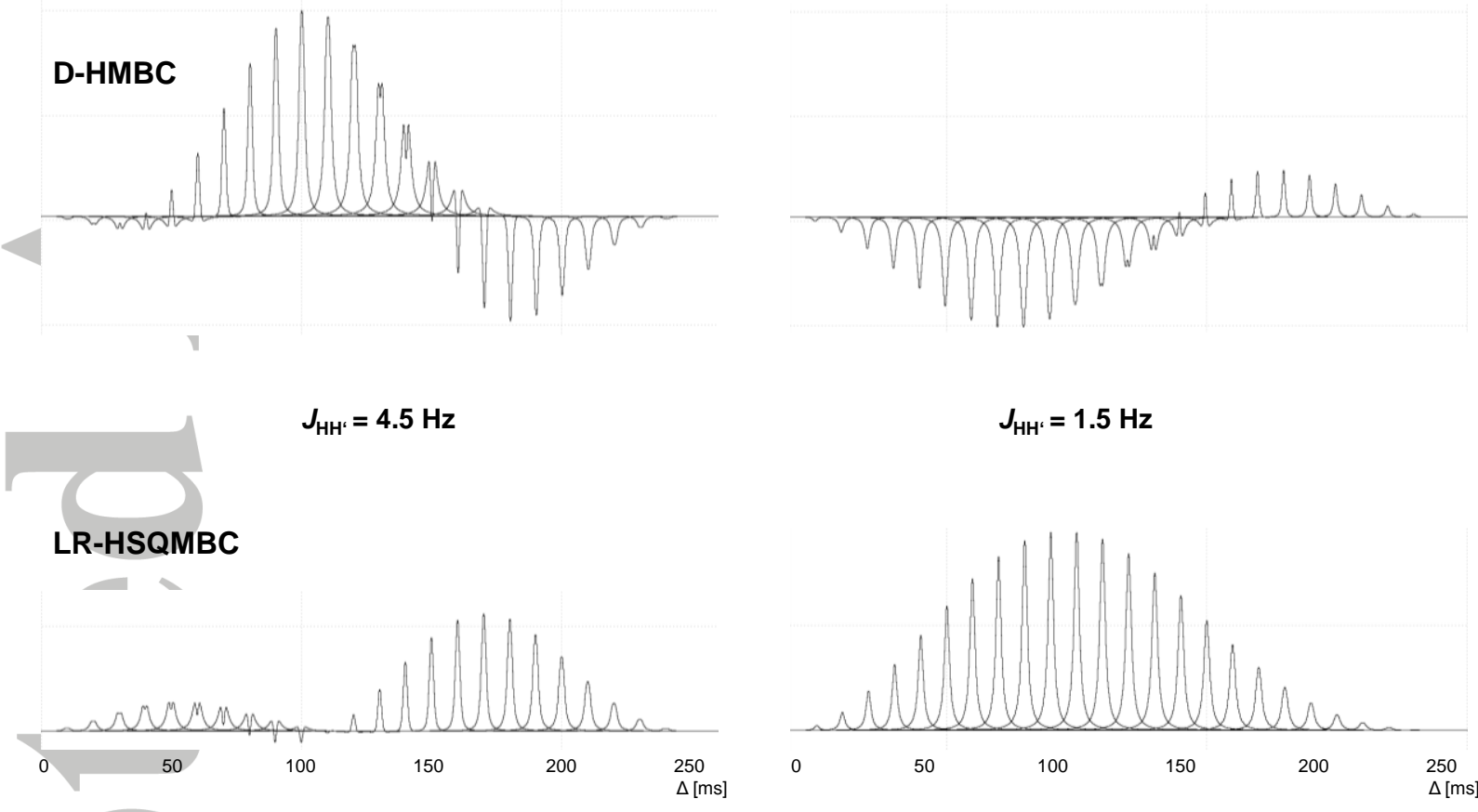

Figure 5. Simulated spectra obtained for a $\mathrm{CH}^{r} \mathrm{H}^{\prime}$ spin system using the LRHSQMBC pulse sequence (bottom) and the D-HMBC pulse sequence (top). The delay $\Delta$ was varied from 0 to $250 \mathrm{~ms}$ in steps of $10 \mathrm{~ms}$. The spectra are displayed in phase sensitive mode. Parameters used: ${ }^{n} J_{C H}=4 \mathrm{~Hz}, J_{H H}=4.5 \mathrm{~Hz}$ (left), $J_{H H}{ }^{\prime}=1.5$ $\mathrm{Hz}$ (right). The relaxation time $\mathrm{T}_{2}$ has been set to $0.4 \mathrm{~s}$ for all nuclei and relaxation during the whole sequence was taken into account. The spectra are displayed at the same signal-to-noise level. Simulations have been performed with the BRUKER NMRSIM program for WINDOWS (version 5.5.3. 2012).

In Figure 5, the predicted minimum in D-HMBC and LR-HSQMBC at $250 \mathrm{~ms}$ $\left(\Delta=1 /{ }^{n} J_{C H}\right)$ is clearly visible, irrespective of the value of the proton-proton coupling constants. The additional predicted zero in LR-HSQMBC for $\mathrm{JHH}^{\prime}=4.5 \mathrm{~Hz}$ at $\sim 110$ ms $\left(\Delta=0.5 / J_{H} H^{\prime}\right)$ is also clearly visible. In contrast, for $J_{H H^{\prime}}=1.5 \mathrm{~Hz}$, an additional 
zero at $\Delta=0.25 / \mathrm{JHH}^{\prime}=167 \mathrm{~ms}$ shows up for D-HMBC. The corresponding zero for the LR-HSQMBC experiment would be at $\Delta=0.5 / \mathrm{JHH}^{\prime}=333 \mathrm{~ms}$, outside the displayed $\Delta$-range.

To study the influence of additional small $J_{H} H^{\prime}$-couplings corresponding simulations with a $\mathrm{CHH}^{\prime}$, a $\mathrm{CHH}^{\prime} \mathrm{H}^{\prime \prime}$, and a $\mathrm{CHH}^{\prime} \mathrm{H}^{\prime \prime} \mathrm{H}^{\prime \prime}$ spin system have been performed (Figure 6)

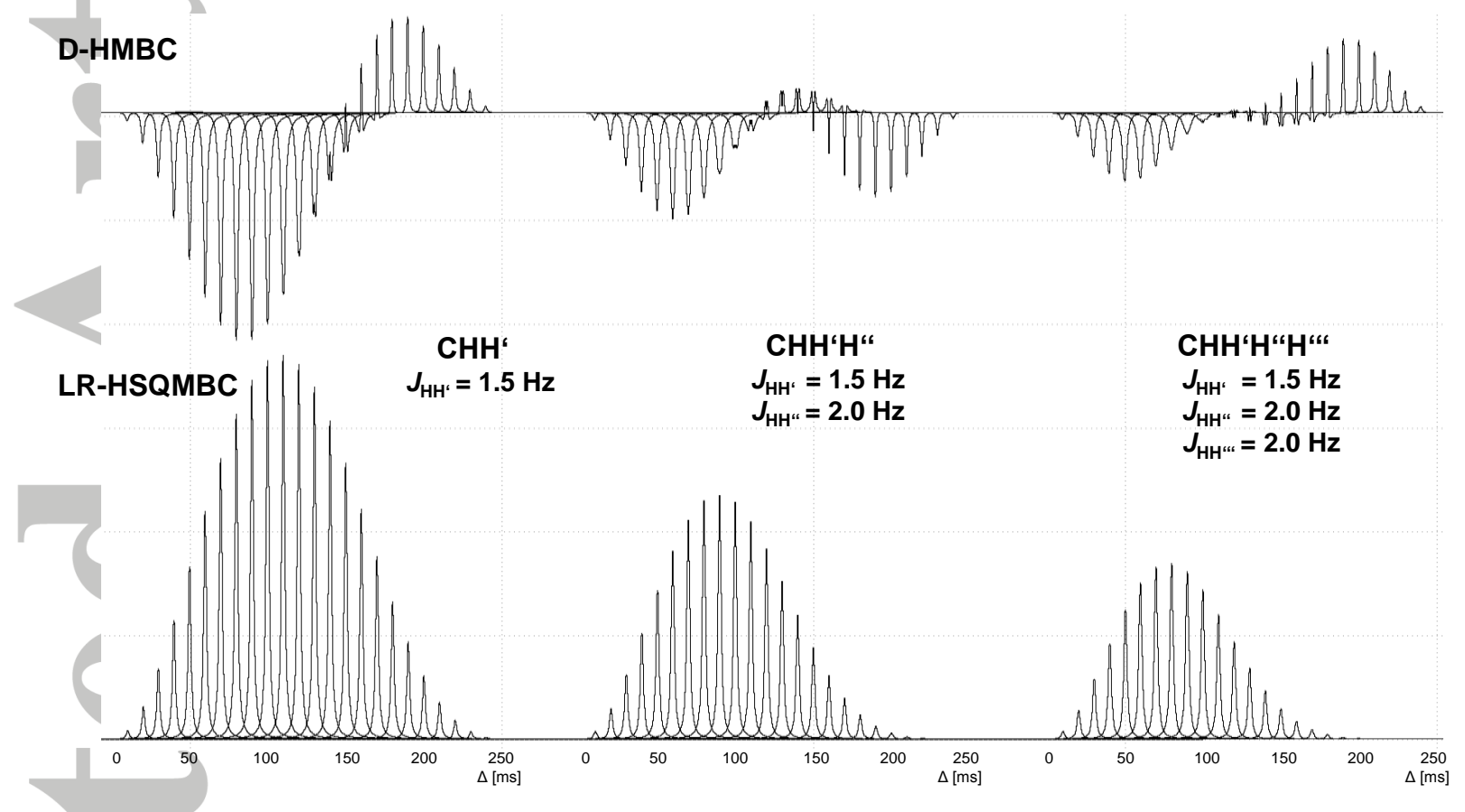

Figure 6. Simulated spectra obtained for a $\mathrm{CH}^{\prime} \mathrm{H}^{\prime}$ spin system (left), a $\mathrm{CH}^{\prime} \mathrm{H}^{\prime} \mathrm{H}^{\prime \prime}$ spin system (middle) and a CH'H'H"H'" spin (right) system using the LR-HSQMBC pulse sequence (bottom) and the D-HMBC pulse sequence (top). The delay $\Delta$ was varied from 0 to $250 \mathrm{~ms}$ in steps of $10 \mathrm{~ms}$. The spectra are displayed in phase sensitive mode. Parameters used: " $J_{\mathrm{CH}}=4 \mathrm{~Hz}, J_{H}{ }^{\prime}=1.5 \mathrm{~Hz}, J_{H H}{ }^{\prime}=J_{H H}{ }^{\prime \prime}=2 \mathrm{~Hz}$. The relaxation time $T_{2}$ has been set to $0.4 \mathrm{~s}$ for all nuclei and relaxation was taken into account during the whole sequence. The spectra are displayed at the same signalto-noise level. Simulations have been performed with the BRUKER NMRSIM program for WINDOWS (version 5.5.3. 2012).

In Figure 6 , the predicted, ${ }^{n} J_{C H}$ triggered minimum in D-HMBC and LRHSQMBC at $\Delta=1 /{ }^{n} J_{C H}=250 \mathrm{~ms}$ is clearly visible, irrespective of the value of the

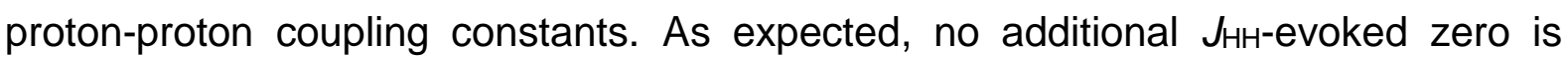


visible with LR-HSQMBC, the first zero occurs at $\Delta=0.5 /{ }^{n} J_{H H}=250 \mathrm{~ms}$. More

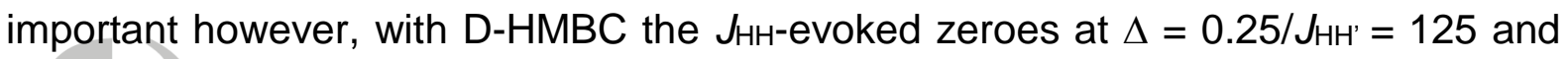
$167 \mathrm{~ms}$ are clearly visible, making the cross peak's intensity very weak within this region compared to LR-HSQMBC. As expected, with an increasing number of weak proton-proton coupling $\mathrm{JHH}_{\mathrm{H}}$ the sensitivity of both experiments generally decrease.

The results of the simulations corroborate the theoretical evaluation: (i) If for the long-range cross peak of interest only large and medium homonuclear protonproton coupling constants $\mathrm{JHH}^{\prime}(>\approx 3 \mathrm{~Hz})$ are involved the intensity of a cross peak in a D-HMBC spectrum is always higher compared to the intensity of this cross peak in a LR-HSQMBC spectrum. This is in agreement with theory (equations 2 \& 4, Table 1) and similar to the results obtained with the non-refocused variants $H M B C$ and LRHSQC. ${ }^{[19]}$ Note that the difference in intensity is maximal for small $\Delta$ values, as noted by Williamson et al., ${ }^{[6]}$ confirming that the LR-HSQMBC is not the best choice for investigating larger heteronuclear coupling constants across 2-3 bonds. (ii) If for the long-range cross peak of interest small proton-proton coupling constants $\mathrm{JHH}^{\prime}(<\approx 3$ $\mathrm{Hz}$ ) are involved the intensity and the number of cross peaks is potentially lower in a D-HMBC- compared to a LR-HSQMBC spectrum. This peculiarity with small $\mathrm{JHH}^{-}$ couplings is caused by the mutual cancelation of antiphase multiplet signals in the $H^{r}-H^{\prime} z$ component. A pure $H^{r}-H^{\prime} z$ component results for D-HMBC at $(0.5 k+0.25) / J_{H H}$, $k=0,1,2, \ldots, n$ and for LR-HSQMBC at $\Delta=(k+0.5) / J_{H H}, k=0,1,2, \ldots, n$ respectively. Whereas these zeroes for the LR-HSQMBC experiment result anyway, irrespective of the size of $\mathrm{JHH}^{\prime}$ (according to theory, see Table 1 and equations 2 \& 4), the zeroes for D-HMBC may appear additionally and - most important - with the first zero at a shorter $\Delta$-value compared to LR-HSQMBC, most likely within the $\Delta^{-}$ range commonly used for detecting very small ${ }^{n} J_{C H}$ couplings.

\subsubsection{Spin systems with homonuclear couplings $\mathrm{JHH}_{\mathrm{H}}>5 \mathrm{~Hz}$ and $\mathrm{J}_{\mathrm{HH}}<5 \mathrm{~Hz}$}

For completeness and to study the effect of homonuclear couplings in the presence of large as well as small coupling constants corresponding simulations with a $\mathrm{CHH} \mathrm{H}^{\prime}$ spin system ( ${ }^{\mathrm{n}} \mathrm{J}_{\mathrm{CH}}=4 \mathrm{~Hz}, \mathrm{JHH}^{\prime}=5 \mathrm{~Hz}$ or $\mathrm{JHH}^{\prime \prime}=1.5 \mathrm{~Hz}, \mathrm{~T}_{2}=0.4 \mathrm{~s}$ for all nuclei) using the LR-HSQMBC and the D-HMBC pulse sequences have been performed (Figure 7). 


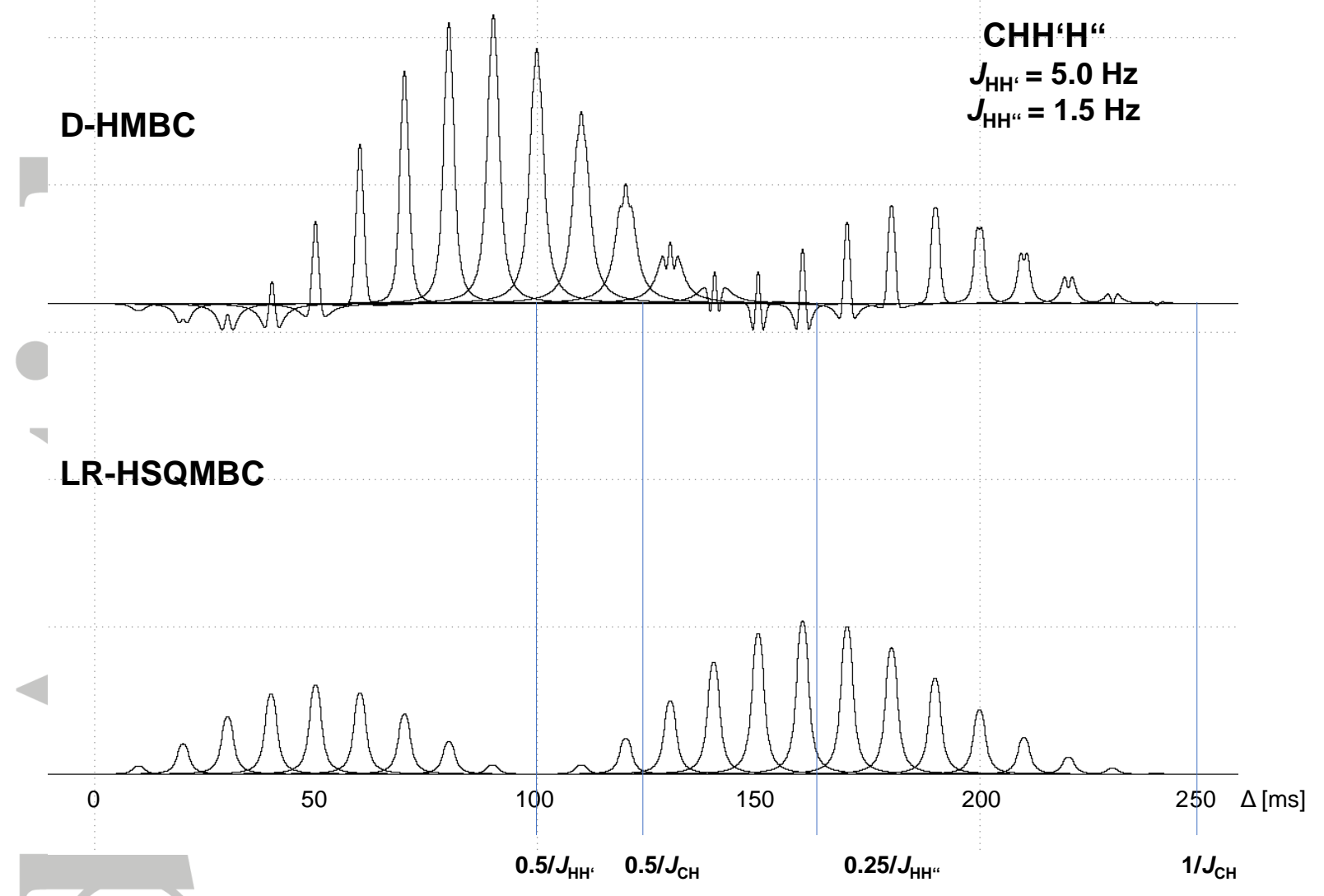

Figure 7. Simulated spectra obtained for a $\mathrm{CH}^{r} \mathrm{H}^{\prime} \mathrm{H}^{\prime}$ " spin system using the LRHSQMBC pulse sequence (bottom) and the D-HMBC pulse sequence (top). The delay $\Delta$ was varied from 0 to $250 \mathrm{~ms}$ in steps of $10 \mathrm{~ms}$. The spectra are displayed in phase sensitive mode. Parameters used: ${ }^{n} J_{\mathrm{CH}}=4 \mathrm{~Hz}, J_{\mathrm{HH}}=5 \mathrm{~Hz}, \mathrm{JHH}^{\prime \prime}=1.5 \mathrm{~Hz}$. The relaxation time $T_{2}$ has been set to $0.4 \mathrm{~s}$ for all nuclei and relaxation during the whole sequence was taken into account. The spectra are displayed at the same signal-tonoise level. Simulations have been performed with the BRUKER NMRSIM program for WINDOWS (version 5.5.3. 2012).

The results of the simulations corroborate the theoretical evaluation: (i) with respect to the overall-sensitivity the D-HMBC experiment is superior in agreement with the theory (equations $2 \& 4$, Table 1 ). This holds in particular for shorter ${ }^{n} J_{C H}$ evolutions delays $(\Delta<100 \mathrm{~ms}$ ) (ii) in agreement with theory zero-intensity occurs at $\Delta=0.5 / \mathrm{JH}^{\prime}(100 \mathrm{~ms})$ for the LR-HSQMBC experiment, caused by the larger homonuclear coupling $\left(\mathrm{JHH}^{\prime}=5 \mathrm{~Hz}\right.$ ) but not for D-HMBC (iii) as expected, weak intensities arise close to $0.25 / \mathrm{JHH}^{\prime}$ exclusively with D-HMBC caused by the small homonuclear coupling $\left(J_{H} H^{\prime}=1.5 \mathrm{~Hz}\right)$ and going along with partial mutual cancelation of closely spaced antiphase multiplet lines. It's interesting to note that due to these 
effects the nominal intensity maximum expected at $\Delta=0.5 / \mathrm{JCH}_{\mathrm{CH}}(125 \mathrm{~ms}$ ) is shifted at lower (D-HMBC) and higher (LR-HSQMBC) delays respectively.

\subsubsection{Effects of relaxation}

As emphasized for a long time, ${ }^{[29]}$ relaxation mechanisms significantly influence the final cross peak's intensity. In the first part of the LR-HSQMBC experiment, $S Q$ relaxation effects are relevant, while in the first part of the D-HMBC, $D Q$ and $Z Q$ effects are effective. ${ }^{[19]}$ Intrinsically, the HMBC experiment is therefore slightly more sensitive when long $\Delta$ values are used and with molecules having shorter relaxation times (Figure S2). [29]

\subsection{Experimental Results}

We utilized strychnine (Figure 8) as the classical model compound to compare the performance of the LR-HSQMBC and the D-HMBC sequences (Figures 9-11 and S3-S8). Strychnine is readily available and has been used as a standard in many NMR studies. ${ }^{[6,15,30-32]}$
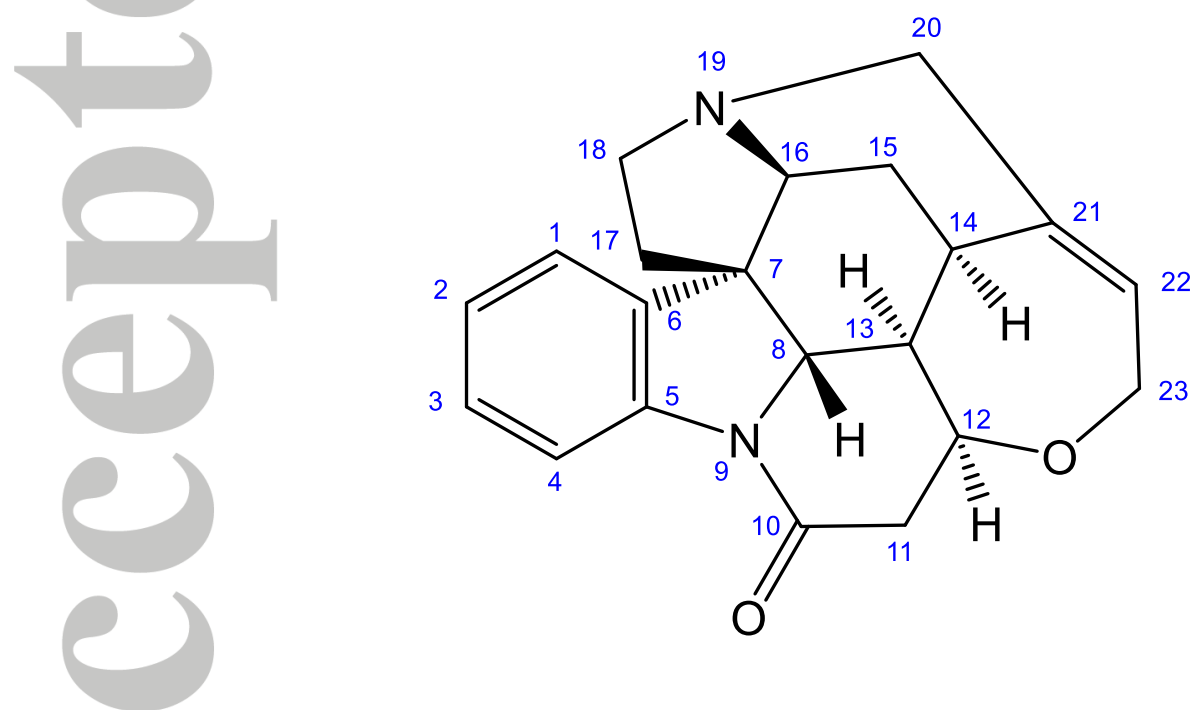

Figure 8. Structure of strychnine and atom numbering.

Although the intensities of corresponding long-range correlation cross peaks are in most cases higher in the D-HMBC spectrum compared to the LR-HSQMBC 
the results are also inconsistent and highly dependent on the number and size of the involved couplings and on the experimental parameters as theoretically predicted. This is corroborated in Figures 10 \& 11, where LR-HSQMBC exhibits long-range correlations that are significantly weaker in the D-HMBC spectrum and vice-versa. For instance, the ${ }^{3} \mathrm{~J}_{\mathrm{C} 7 \mathrm{H} 13}$ cross peak is clearly visible in the LR-HSQMBC spectrum, while it remains invisible in the D-HMBC spectrum (Figure S4). The corresponding 1D rows extracted at a ${ }^{13} \mathrm{C}$ chemical shift $\delta=52.1 \mathrm{ppm}(\mathrm{C} 7)$ (Figure S5), show that the ${ }^{3} \mathrm{~J}_{\mathrm{C} 7 \mathrm{H} 13}$ cross peak exists but is weak in the D-HMBC spectrum. On the other hand, all other correlations are markedly more intense. In contrast, the ${ }^{3} \mathrm{~J}_{\mathrm{C} 21 \mathrm{H} 15 \mathrm{a}}$ and ${ }^{3} J_{\mathrm{C} 21 \mathrm{H} 13}$ cross peaks are clearly visible in the D-HMBC spectrum, while they are very weak in the LR-HSQMBC spectrum (Figure 10).

Focusing on long-range correlations across more than 4 bonds, weak ${ }^{4} J_{66 H 13}$ and ${ }^{4} J_{C 5 H 13}$ cross peaks are visible in the LR-HSQMBC- but not in the D-HMBC spectrum, while a weak ${ }^{4} \mathrm{~J}_{\mathrm{C} 6 \mathrm{H} 15 \mathrm{a}}$ cross peak is visible in the D-HMBC- but not in the LR-HSQMBC spectrum (Figure 10). The corresponding 1D rows extracted from the D-HMBC and LR-HSQMBC spectra are shown in Figures 11 and S6-S7, and other examples can be found in the Supplementary Information (Figures S8-S9). It can be for instance seen that weak ${ }^{5} \mathrm{~J}_{\mathrm{C} 6 \mathrm{H} 11 \mathrm{a}},{ }^{4} \mathrm{~J}_{\mathrm{C} 6 \mathrm{H} 15 \mathrm{a}}$, and ${ }^{4} \mathrm{~J}_{\mathrm{C} 6 \mathrm{H} 18 \mathrm{~b}}$ cross peaks are visible in the D-HMBC spectrum, but not in the LR-HSQMBC spectrum (Figure S7).

D-HMBC and LR-HSQMBC simulated spectra obtained for the spin system $\mathrm{C}_{21} \mathrm{H}_{8} \mathrm{H}_{12} \mathrm{H}_{13} \mathrm{H}_{14}\left({ }^{3} \mathrm{~J}_{21 \mathrm{H} 13}=1.4 \mathrm{~Hz},{ }^{3} \mathrm{HH}_{\mathrm{H} 13}=10.5 \mathrm{~Hz},{ }^{3} \mathrm{H}_{\mathrm{H} 12 \mathrm{H} 13}=3.2 \mathrm{~Hz},{ }^{3} \mathrm{H}_{\mathrm{H} 13 \mathrm{H} 14}=3.2\right.$ $\mathrm{Hz}$ ) shown in Figure S10 fairly support our experimental observation: for $\Delta=166 \mathrm{~ms}$, the ${ }^{3} \mathrm{~J}_{\mathrm{C} 21 \mathrm{H} 13}$ is predicted to be intense in the D-HMBC spectrum, and virtually missing in the LR-HSQMBC spectrum. A tentative explanation for the discrepancy between the theoretical and experimental results is that additional small long range protonproton couplings such as ${ }^{4} \mathrm{~J}_{\mathrm{H} 13 \mathrm{H} 11 \mathrm{a}, \mathrm{b}}$ or ${ }^{4} \mathrm{~J}_{\mathrm{H} 13 \mathrm{H} 15 \mathrm{a}, \mathrm{b}}$, not visible in the ${ }^{1} \mathrm{H}$ - or COSY spectra, could contribute and influence the intensity of the cross peaks, as discussed in the theory.

Intriguingly, and addressing the performance of low-pass $J$ filtering last, the classical low-pass $J$ filter implemented in the D-HMBC seems to be more efficient than the G-BIRD ${ }^{r}, X$ element implemented in the LR-HSQMBC, as shown by Figure S11. This is somewhat surprising as for the non-decoupled variants the G-BIRD element performs better than the low-pass $J$ filter, unless higher orders of the $J$-filter 
are used. ${ }^{[14,33-35]}$ A breakthrough of ${ }^{1} \mathrm{~J} \mathrm{CH}$ cross peaks in spectra obtained with the non-refocused HMBC experiment without ${ }^{13} \mathrm{C}$ broadband decoupling is usually not misleading. Corresponding residual peaks can be readily identified thanks to their widely split doublets and can also be used for signal assignment. [14] However, their occurrence in decoupled experiments like the D-HMBC and LR-HSQMBC can be more troublesome, and can lead to misinterpretation. The implementation of a double-tuned G-BIRD filter in the LR-HSQMBC experiment might be considered for efficiently removing residual ${ }^{1} \mathrm{~J}_{\mathrm{CH}}$ cross peaks. ${ }^{[35]}$

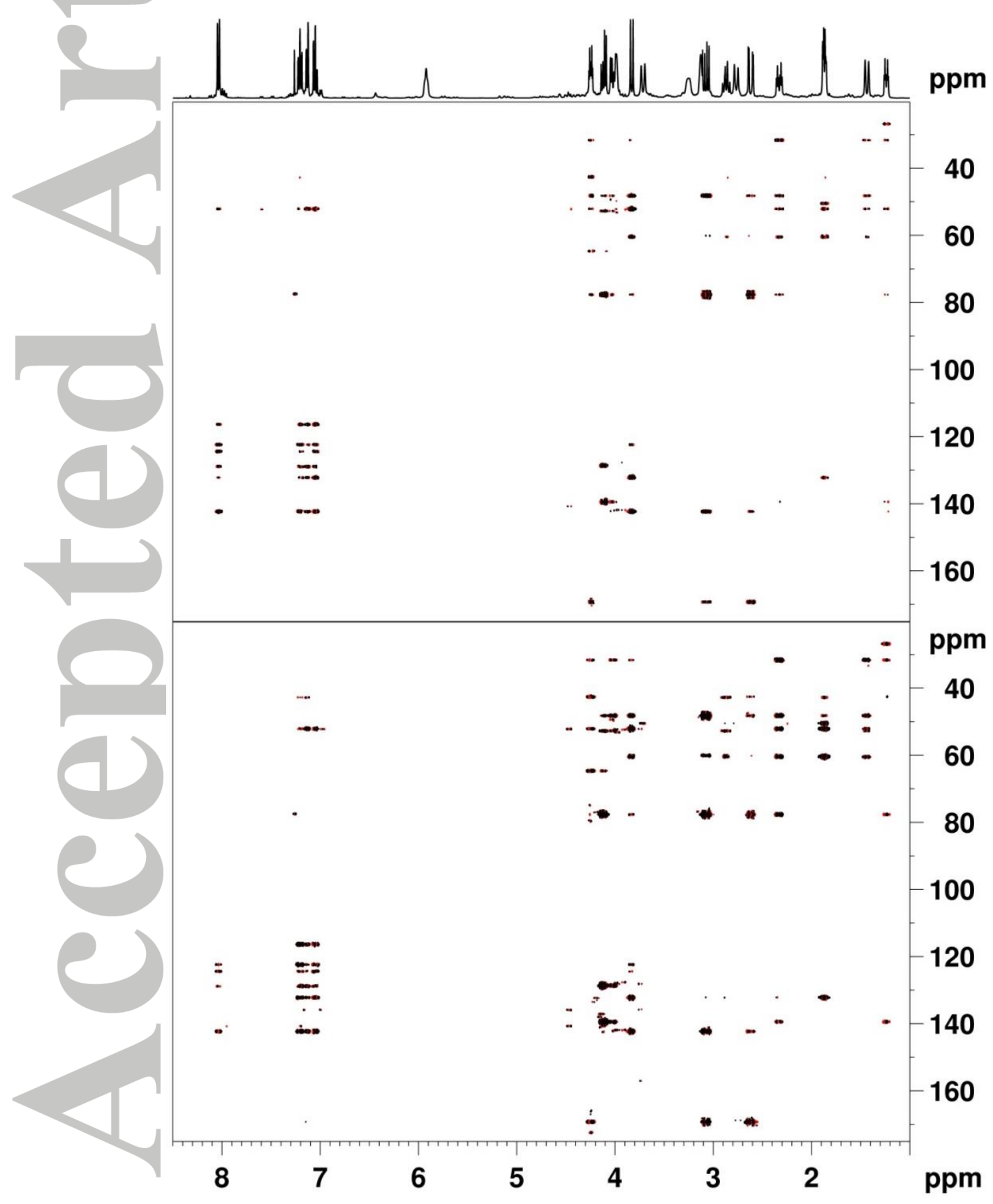


Figure 9. D-HMBC (bottom) and LR-HSQMBC spectra (top) optimized for ${ }^{n} J_{\mathrm{CH}}=3$ $\mathrm{Hz}\left(\Delta=0.5 /{ }^{n} J_{\mathrm{CH}}=166 \mathrm{~ms}\right)$. Both data were acquired using a $30 \mathrm{mg}$ sample of strychnine dissolved in $0.6 \mathrm{~mL}$ of $\mathrm{CDCl}_{3} .768 \times 2048$ data points were acquired in $t_{1}$ and $t_{2}$ respectively with 32 transients accumulated per $t_{1}$ increment. The delay $\tau$ for suppressing one-bond signals was set to $3.45 \mathrm{~ms}$. The duration of each gradient was $\delta=1 \mathrm{~ms}$, the gradient recovery was $0.2 \mathrm{~s}$. The acquisition time was $0.214 \mathrm{~s}$, the spectral width was $4795 \mathrm{~Hz}$ in F2 and $15596 \mathrm{~Hz}$ in F1; the relaxation delay was $1 \mathrm{~s}$, for a total experimental time of approximately $8 \mathrm{~h}$. Prior to Fourier transformation, zero filling to 4096 points in $\mathrm{F} 2,1024$ points in $\mathrm{F} 1$, and weighting with a sine squared function were applied in both dimensions. The spectra are displayed at the same noise level and in phased mode with respect to F2.

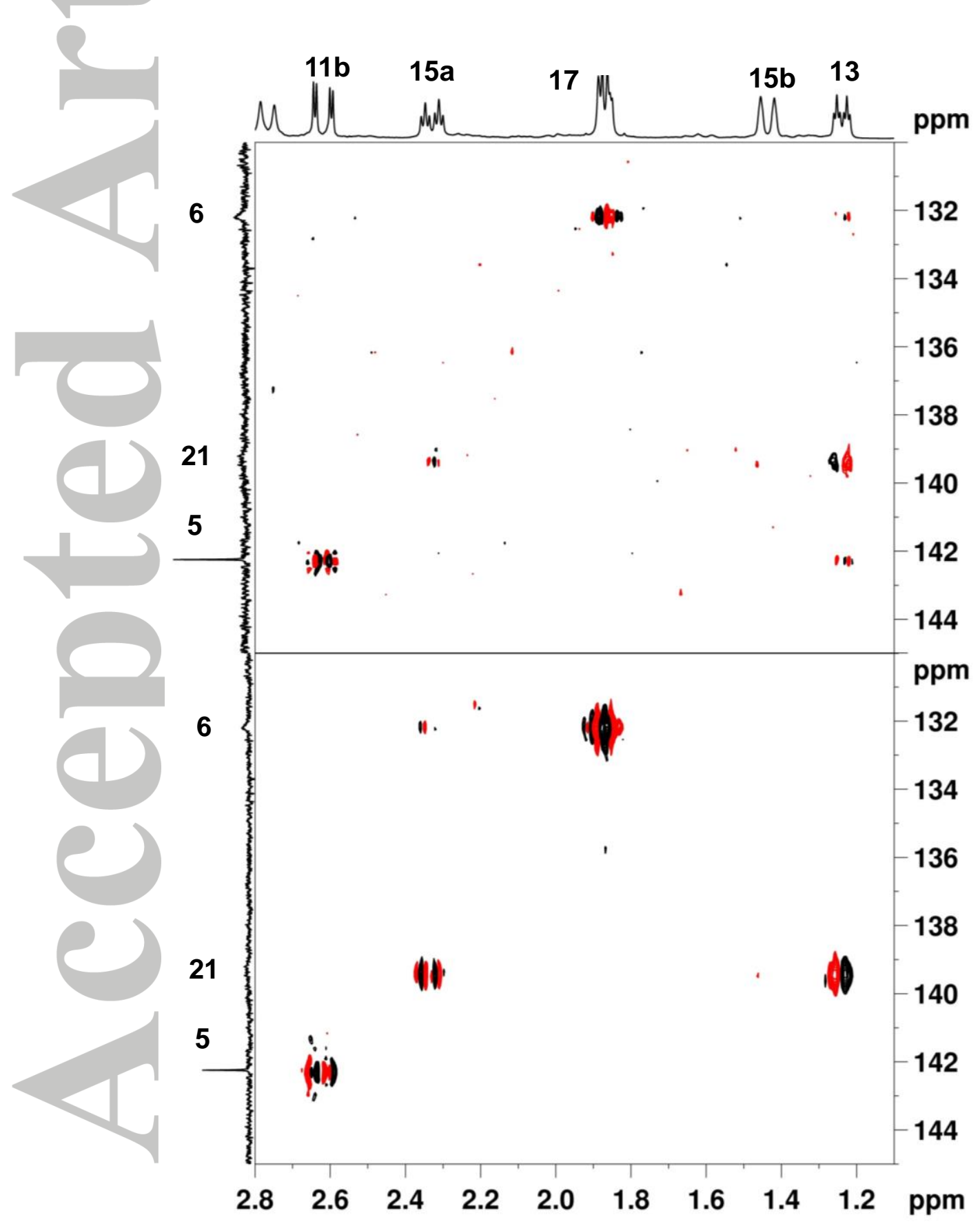

This article is protected by copyright. All rights reserved. 
Figure 10. Expansions of the D-HMBC (bottom) and LR-HSQMBC spectra (top) optimized for ${ }^{n} J_{C H}=3 \mathrm{~Hz}\left(\Delta=0.5 /{ }^{n} J_{C H}=166 \mathrm{~ms}\right)$ showing the long-range correlations of $\mathrm{H} 11 \mathrm{~b}, \mathrm{H} 15 \mathrm{a}, \mathrm{H} 17$ and $\mathrm{H} 13$ with $\mathrm{C} 5, \mathrm{C} 6$ and $\mathrm{C} 21$, respectively. The spectra are displayed at the same noise level and in phased mode with respect to F2.

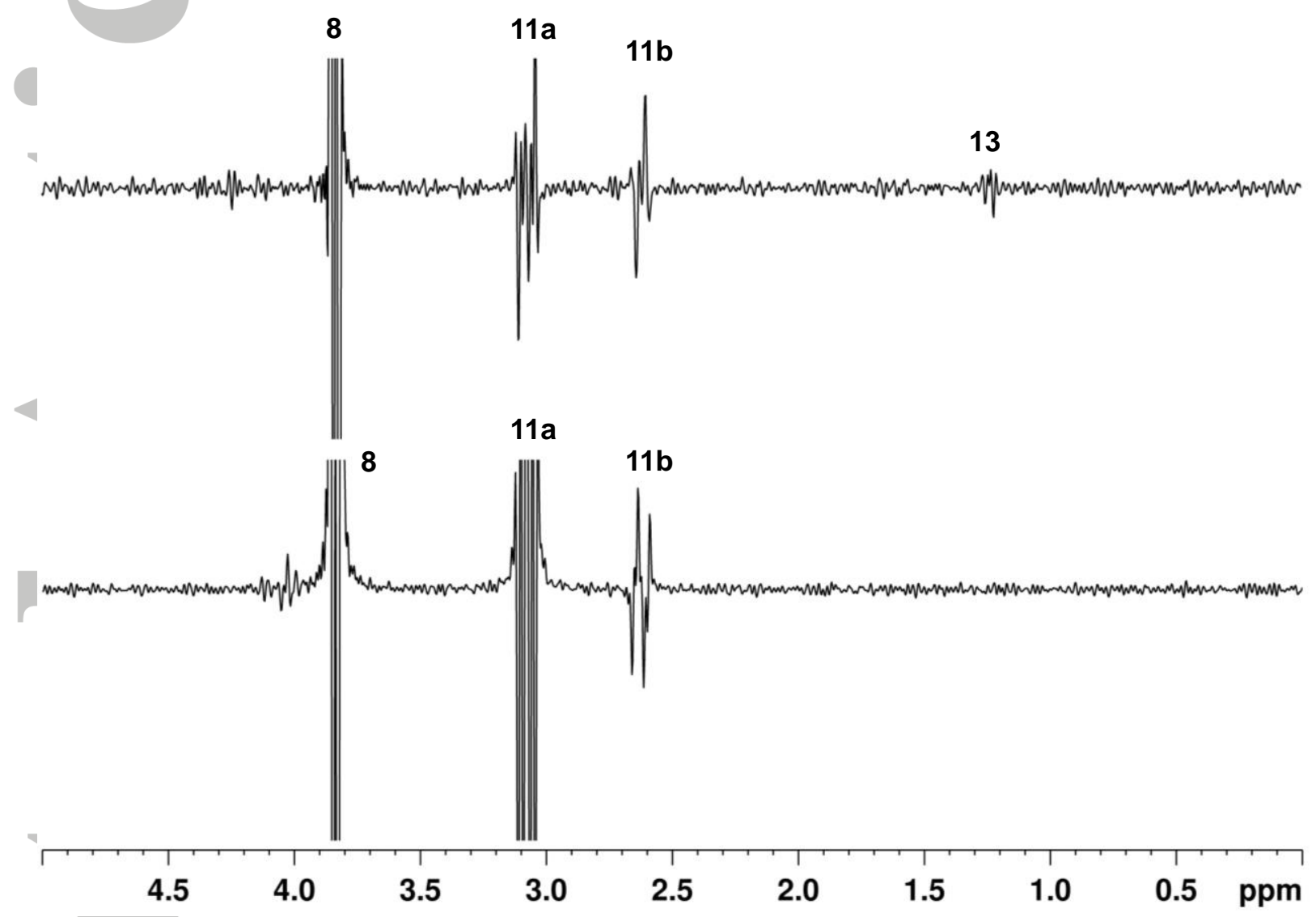

Figure 11. $1 \mathrm{D}$ rows extracted from the $3 \mathrm{~Hz}$ optimized D-HMBC (bottom) and LRHSQMBC (top) spectra of strychnine dissolved in $\mathrm{CDCl}_{3}$ showing the long-range correlations associated with the $C 5$ resonance $(\delta=142.2 \mathrm{ppm})$. Both spectra are displayed with the same noise level.

\section{Conclusion}

In this contribution and based on our previous results obtained with the nondecoupled variants HMBC and LR-HSQC[19], we have analyzed their refocused counterparts D-HMBC and LR-HSQMBC with final ${ }^{13} \mathrm{C}$ broadband decoupling both theoretically and experimentally and with the focus on the influence of homonuclear couplings $\mathrm{JHH}^{\prime}$. Similar to the non-refocused variants $\mathrm{HMBC}$ and LR-HSQC, ${ }^{[19]}$ it turns 
out that the same two observable multiplet components are present before acquisition with D-HMBC and LR-HSQMBC. Yet, while the numerical coefficient of the in-phase $\left(H_{-}\right)$multiplets is identical in both experiments, the numerical coefficient of the anti-phase $\left(H_{.} H_{z}^{\prime}\right)$ multiplets is two times as large for D-HMBC compared to LR-HSQMBC. Furthermore, additional intensity-zeroes caused by $J_{H H}$-couplings occur with LR-HSQMBC at $\Delta=(0.5+k) / J_{H H}$ (with $k=0,1,2, \ldots, n$ ). Consequently and with medium to large homonuclear couplings $\mathrm{JHH}_{\mathrm{H}}$, i.e. couplings producing wellresolved multiplet lines $\left(\mathrm{JHH}_{\mathrm{H}}>3 \mathrm{~Hz}\right)$, the intensity of the long-range cross peaks is generally higher in D-HMBC spectra.

However, when very small homonuclear couplings $\mathrm{JHH}^{\prime}$ are involved, typically more cross-peaks are visible in a LR-HSQMBC spectrum compared to a D-HMBC spectrum. This must be attributed to the antiphase component $H_{-} H_{Z}^{\prime}$ : its intensity may be decreased or it even may vanish due to mutual cancelation of the closely spaced antiphase multiplet lines. A pure $H_{.} H^{\prime}$ z component, hence weakened (or even canceled) cross-peaks arise at $\Delta=(0.5 k+0.25) / J_{H H}, k=0,1,2, \ldots, n$, with the DHMBC experiment. Consequently, a better sensitivity, allowing for detecting also weak ${ }^{n} J_{C H}$-interactions are expected to result with the LR-HSQMBC experiment, whereas blind $\Delta$-regions due to small $\mathrm{JHH}_{\mathrm{H}}$ couplings may occur with D-HMBC.

The different behavior of the LR-HSQMBC and the D-HMBC experiment with respect to homonuclear couplings $J_{H H}$ may be considered also concentrating on heteronuclear couplings ${ }^{n} J_{C H}$, the central parameter with these experiments. Scientists, focused on the detection of heteronuclear long-range interactions have to decide whether to detect medium-to-large $(\mathrm{J}>3 \mathrm{~Hz})$ or small ${ }^{n} \mathrm{~J}_{\mathrm{CH}}$ interactions $(\mathrm{J}<3$ $\mathrm{Hz}$ ). Hence they should be aware of the corresponding characteristics of the LRHSQMBC and D-HMBC: With the experimental setup directed to the detection of medium-to-large ${ }^{n} J_{C H}$ interactions and with the intention to keep relaxation losses small, i.e. by choosing a short delay $\Delta$ (e.g. $50 \mathrm{~ms}$ ), the D-HMBC experiment will be superior as discussed before, irrespective of the number and size of homonuclear couplings since the unfavorable peculiarity with small $\mathrm{JHH}_{\mathrm{H}}$-couplings, showing up only at larger $\Delta$-delays, will rarely become manifest in the D-HMBC spectrum. However, with the experimental setup arranged for detecting small ${ }^{n} J_{C H}$-interactions, hence by choosing a relatively long delay $\Delta$ (e.g. $200 \mathrm{~ms}$ ), the performance of the DHMBC experiment will be hampered by a sensitivity decrease caused by small $\mathrm{JHH}_{\mathrm{H}}$ - 
couplings. Hence and despite the existing intensity zeroes caused by $\mathrm{JHH}^{\prime}$ of any size at $\Delta=(0.5+k) / J_{H H^{\prime}}$ the LR-HSQMBC experiment may be the better choice in such a case.

Another central difference resides in the design of both pulse sequences: in HMBC-based sequences, homonuclear $\mathrm{JHH}_{\mathrm{H}}$ couplings evolve during the entire $t_{1}$ evolution period, giving tilted multiplet structures along the $F_{1}$ dimension, that in turn significantly reduces the sensitivity which is not the case of HSQC-based experiments.

We and other groups found in experimental investigations that with the focus on the detection of weak ${ }^{n} J_{C H}$-interactions the LR-HSQMBC performs indeed better than the D-HMBC with respect to the number and intensity of cross-peak attributed to weak ${ }^{n} J_{C H}$ correlations, provided a sufficient amount of $t_{1}$ increments $\left(n t_{1}>512\right)$ are acquired in the $\mathrm{F} 1$ dimension. ${ }^{[6]}$ On a routine level, we therefore recommend running both experiments tuned for large (D-HMBC) and small (LR-HSQMBC) ${ }^{n} J_{\mathrm{CH}}$, since a priori one may not know the spin systems under study.

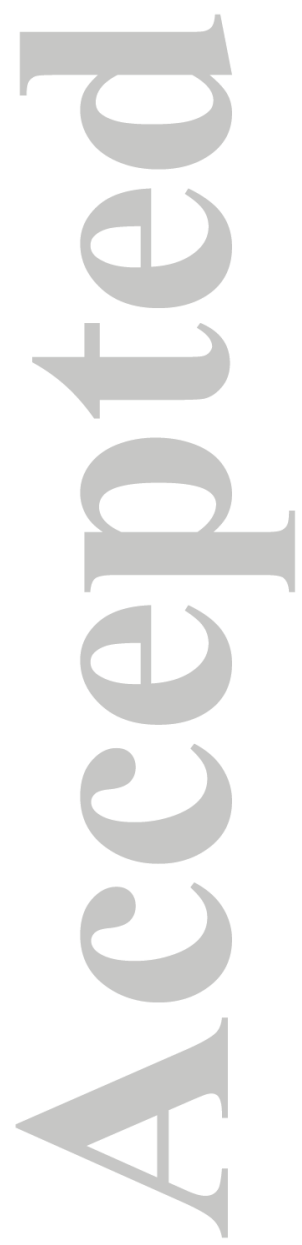




\section{References}

[1] G. E. Martin, R. C. Crouch, J. Nat. Prod. 1991, 54, 1-70.

[2] G. E. Martin, C. E. Hadden, J. Nat. Prod. 2000, 63, 543-585.

[3] G. E. Martin, A. S. Zektzer, Magn. Reson. Chem. 1988, 26, 631-652.

[4] A. Bax, M. F. Summers, J. Am. Chem. Soc. 1986, 108, 2093-2094.

[5] J. Furrer, Concepts Magn. Reson., Part A 2012, 40A, 101-127.

[6] R. T. Williamson, A. V. Buevich, G. E. Martin, T. Parella, J. Org. Chem. 2014, 79, 3887-3894.

[7] J. Sauri, G. E. Martin, in Modern NMR Approaches to the Structure Elucidation of Natural Products: Volume 2: Data Acquisition and Applications to Compound Classes, Vol. 2, The Royal Society of Chemistry, 2017, pp. 315-357.

[8] R. Araya-Maturana, T. Delgado-Castro, W. Cardona, B. E. Weiss-Lopez, Curr. Org. Chem. 2001, 5, 253-263.

[9] M. Köck, B. Reif, W. Fenical, C. Griesinger, Tetrahedron Lett. 1996, 37, 363-366.

[10] B. Reif, M. Köck, R. Kerssebaum, H. Kang, W. Fenical, C. Griesinger, J. Magn. Reson. 1996, 118, 282-285.

[11] G. E. Martin, in Annu. Rep. NMR Spectrosc., Vol. 74 (Ed.: G. A. Webb), Academic Press, 2011, pp. 215-291.

[12] M. M. Senior, R. T. Williamson, G. E. Martin, J. Nat. Prod. 2013, 76, 2088-2093.

[13] K. Furihata, H. Seto, Tetrahedron Lett. 1995, 36, 2817-2820.

[14] J. Furrer, Concepts Magn. Reson., Part A 2012, 40A, 146-169.

[15] T. Parella, J. F. Espinosa, Prog. Nucl. Magn. Reson. Spectrosc. 2013, 73, 1755.

[16] K. E. Kover, G. Batta, K. Feher, J. Magn. Reson. 2006, 181, 89-97.

[17] S. Boros, K. E. Kover, Magn. Reson. Chem. 2011, 49, 106-110.

[18] S. Gil, J. F. Espinosa, T. Parella, J. Magn. Reson. 2011, 213, 145-150.

[19] P. Bigler, J. Furrer, Magn. Reson. Chem. 2018, 56, 1101-1116.

[20] S. Heikkinen, I. Kilpeläinen, J. Magn. Reson. 1999, 137, 93-99.

[21] B. L. Marquez, W. H. Gerwick, R. T. Williamson, Magn. Reson. Chem. 2001, 39, 499-530.

[22] G. E. Martin, private communication 2018.

[23] J. M. Bohlen, G. Bodenhausen, J. Magn. Reson. 1993, 102, 293-301. 
[24] C. Emetarom, T. L. Hwang, G. Mackin, A. J. Shaka, J. Magn. Reson. 1995, 115, 137-140.

[25] R. T. Williamson, A. V. Buevich, G. E. Martin, Tetrahedron Lett. 2014, 55, 33653366.

[26] D. O. Cicero, G. Barbato, R. Bazzo, J. Magn. Reson. 2001, 148, 209-213.

[27] A. Meissner, O. W. Sorensen, Magn. Reson. Chem. 2001, 39, 49-52.

[28] H. Kogler, O. W. Sørensen, G. Bodenhausen, R. R. Ernst, J. Magn. Reson. 1983, 55, 157-163.

[29] R. R. Ernst, G. Bodenhausen, A. Wokaun, Principles of nuclear magnetic resonance in one and two dimensions, Clarendon Press, Oxford, 1987.

[30] J. Furrer, Concepts Magn. Reson., Part A 2015, 43, 177-206.

[31] J. Sauri, G. E. Martin, J. Furrer, Concepts Magn. Reson., Part A 2015, 44A, 227251.

[32] P. Bigler, J. Furrer, Magn. Reson. Chem. 2018, 56, 329-337.

[33] R. Burger, C. Schorn, P. Bigler, Magn. Reson. Chem. 2000, 38, 963-969.

[34] J. Furrer, Magn. Reson. Chem. 2006, 44, 845-850.

[35] J. Furrer, D. Thévenet, Magn. Reson. Chem. 2009, 47, 239-248. 

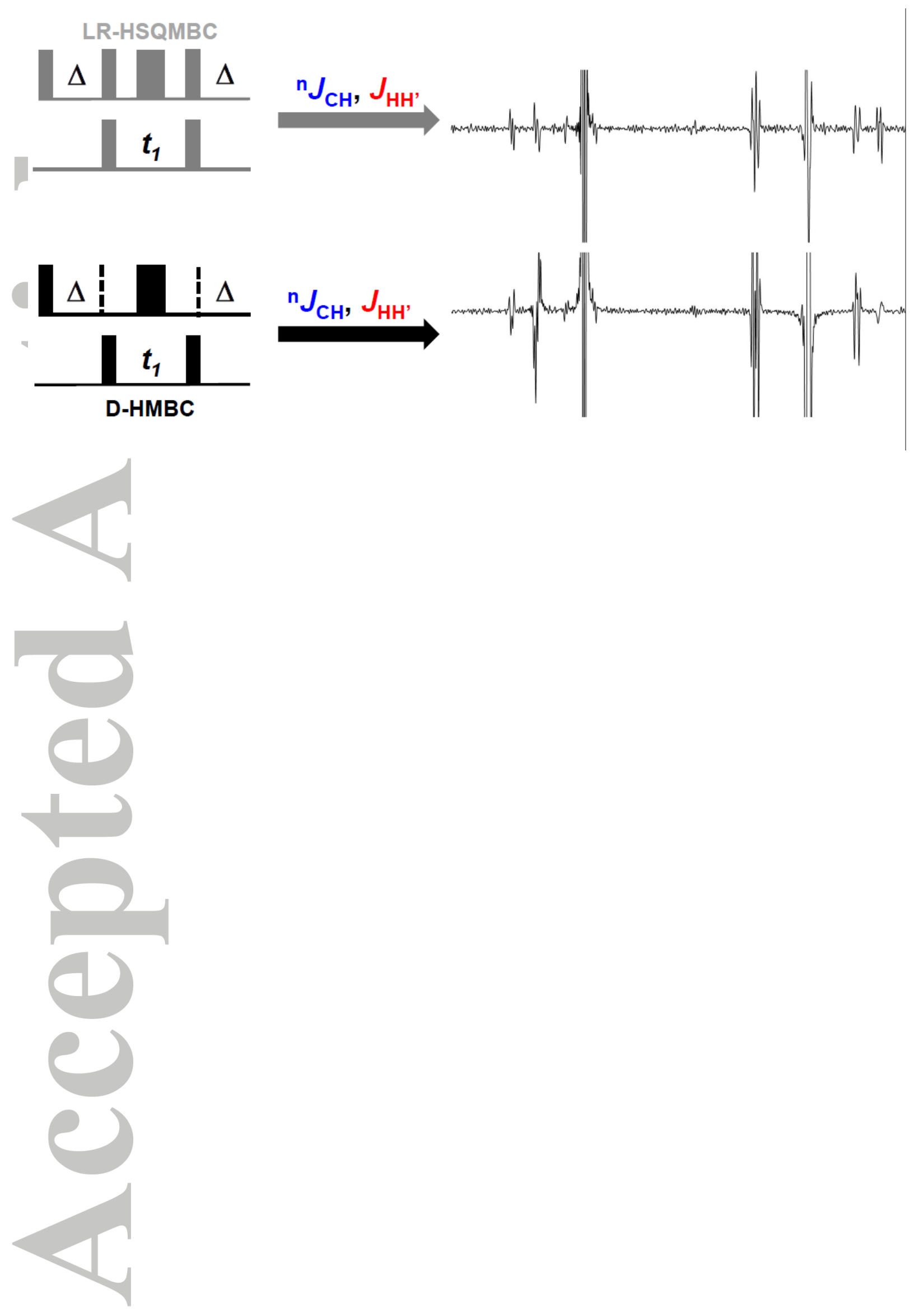